NASA Contractor Report 194945

ICASE Report No. 94-56

NASA-CR-194945

19940033095
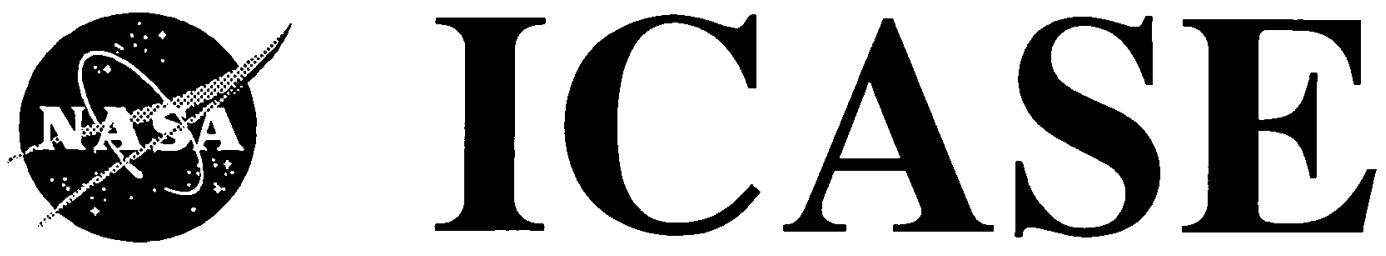

\title{
TOWARDS ENHANCING AND DELAYING DISTURBANCES IN FREE SHEAR FLOWS
}

\section{W.O. Criminale}

\section{T.L. Jackson}

D.G. Lasseigne

Contract NAS1-19480

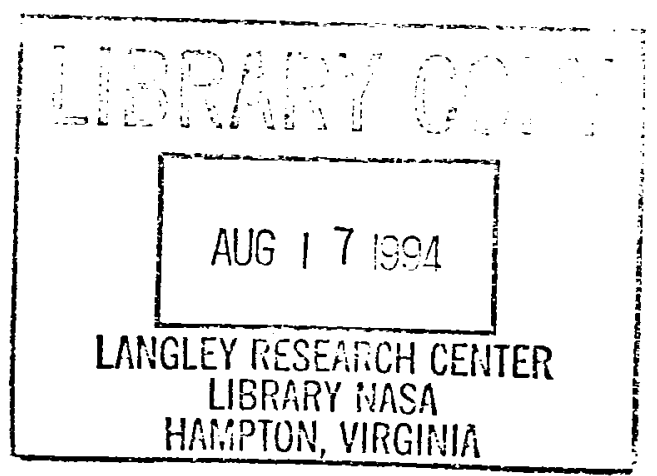

June 1994

Institute for Computer Applications in Science and Engineering NASA Langley Research Center

Hampton, VA 23681-0001

(SRA Operated by Universities Space Research Association 



\section{ICASE Fluid Mechanics}

Due to increasing research being conducted at ICASE in the field of fluid mechanics, future ICASE reports in this area of research will be printed with a green cover. Applied and numerical mathematics reports will have the familiar blue cover, while computer science reports will have yellow covers. In all other aspects the reports will remain the same; in particular, they will continue to be submitted to the appropriate journals or conferences for formal publication. 



\title{
TOWARDS ENHANCING AND DELAYING DISTURBANCES IN FREE SHEAR FLOWS ${ }^{1}$
}

\author{
W.O. Criminale \\ Department of Applied Mathematics \\ University of Washington \\ Seattle, Washington 98195 \\ T.L. Jackson \\ Institute for Computer Applications in Science and Engineering \\ NASA Langley Research Center \\ Hampton, VA 23681-0001 \\ and \\ D.G. Lasseigne \\ Department of Mathematics and Statistics \\ Old Dominion University \\ Norfolk, Virginia 23529
}

\begin{abstract}
The family of shear flows comprising the jet, wake, and the mixing layer are subjected to perturbations in an inviscid, incompressible fluid. By modeling the basic mean flows as parallel with piecewise linear variations for the velocities, complete and general solutions to the linearized equations of motion can be obtained in closed form as functions of all space variables and time when posed as an initial-value problem. The results show that there is a continuous as well as the discrete spectrum that is more familiar in stability theory and therefore there can be both algebraic and exponential growth of disturbances in time. These bases make it feasible to consider control of such flows. To this end, the possibility of enhancing the disturbances in the mixing layer and delaying the onset in the jet and wake is investigated. It is found that growth of perturbations can be delayed to a considerable degree for the jet and the wake but, by comparison, cannot be enhanced in the mixing layer. By using moving coordinates, a method for demonstrating the predominant early and long time behavior of disturbances in these flows is given for continuous velocity profiles. It is shown that the early time transients are always algebraic whereas the asymptotic limit is that of an exponential normal mode. Numerical treatment of the new governing equations confirm the conclusions reached by use of the piecewise linear basic models. Although not persued here, feedback mechanisms designed for control of the flow could be devised using the results of this work.
\end{abstract}

\footnotetext{
${ }^{1}$ This work was supported by the National Aeronautics and Space Administration under NASA Contract No. NAS1-19480 while in residence at the Institute for Computer Applications in Science and Engineering (ICASE), NASA Langley Research, Hampton, VA 23681-0001.
} 



\section{INTRODUCTION}

Classical hydrodynamic stability theory has been successful in demonstrating how any particular shear flow must be unstable. For the class of flows where the fluid can be taken as inviscid there are a variety of results from both theoretical and experimental investigations. Indeed, the corroboration between the two pursuits is quite strong and has led to a reasonable understanding of the problems. There are, however, still significant needs. The determination of whether or not a flow is stable or unstable has been ascertained by the use of normal mode or traveling wave analysis for the linearized equations of motion. This technique is tantamount to computing a discrete spectrum of eigenvalues. Unfortunately, even this approach has been limited in that the effort has concentrated on determining if there is at least one unstable eigenvalue. The remaining eigenvalues (persumbly infinite in number), including all those that are stable, must be known in order to consider any initial-value problem. In addition, the continuous spectrum, if it exists, must also be included for any general specification. The determination of an unstable eigenvalue only ignores transient dynamics in favor of the asymptotic state. In other words, the flow is unstable regardless of how perturbations are imparted. If one desires to understand how to influence any shear flow, it is clear that the origin and the transients must be examined. Traditional means of solution of the governing equations where a separable normal mode traveling wave is introduced is not a profitable means of accomplishing this task. The difficulties lie with the fact that the principal equation (Rayleigh equation) used in the inviscid problem is not self adjoint and therefore does not allow classical Sturm-Liouville means for calculations. To include three-dimensional effects requires solving yet another equation (Squire mode equation) and it too is not of standard form. Including a viscous fluid in the investigation (requiring the Orr-Sommerfeld equation) could lead to a singular perturbation problem, making for still more complications. The question, then, is how to obtain a reasonable solution for a workable model that will permit a general treatment of initial-values.

The class of free shear flows that occur without the influence of solid boundaries can be analyzed in an inviscid fluid because, unlike the boundary layer prototype, viscous effects tend to cause damping rather than instability, i.e., any eigenvalue is decreased in magnitude by viscosity. The inviscid fluid approximation allows for the removal of one of the major complications cited above. In addition, it permits the analysis to be made with a parallel flow assumption. An introduction of separable solutions for all the dependent variables in the form of traveling waves usually follows. This procedure is fine so long as every eigenvalue and eigenfunction are known, particularly the ones that are damped since this information is crucial to the transient dynamics. The continuous spectrum remains omitted with this type of solution.

An alternative approach to the initial-value problem is that due to Kelvin (1887) and later by Orr $(1907 \mathrm{a}, 1907 \mathrm{~b})$. This method involves a change of the independent variables from a Cartesian to a moving frame. As a result, in this frame, there is no critical layer that has so complicated normal mode calculations. The method can be used for any profile but is most amenable to ones that are piecewise linear. The modeling of the mean velocity in this manner in no way loses any of the important qualitative information and, it will be seen, all of the early and asymptotic temporal behavior can be captured. The fact that a growing normal mode occurs for small values of the wave number (large scales when compared to the scale of the flow field) is also where the piecewise linear profile is best valid. Of course, this approximation is not without its negative aspects (only a finite number of normal modes are possible, e.g.) but, the fact that the problems have also been done numerically when cast in terms of normal modes, 
any misinformation from this part of the solution can be eliminated by comparison. In short, the advantage gained outweighs the difficulties and this will be demonstrated.

Work that has been done by direct numerical simulation of these flows using the full NavierStokes equations usually introduces the most amplified normal mode to initiate the dynamics. Experimentalists have used various devices to attempt to enhance or delay disturbances based on the normal mode frequencies. Neither of these studies was concerned with how any disturbances had their origins. In a somewhat general way, it is assumed that the standard linear theory provides this data. In the case of the mixing layer prototype, Bun and Criminale (1994) have shown that this reliance can be misleading. In fact, this work demonstrated that no growing normal mode is needed at all to achieve the well-known roll up process that is observed in this flow. It was possible to do this because the origin and the transient temporal dynamics are known explicitly. The same procedure can be used for the jet and wake family. The natural query for any case is whether or not this part of the flow can be manipulated in such a way as to enhance or delay perturbations as may be desired. This topic is the central issue of this paper.

\section{BASIC GOVERNING EQUATIONS}

The fluid is taken as inviscid and incompressible with the basic flow parallel, $U=U(y), V=$ $W=0$. Then, the linearized equations of motion can be written as

$$
\begin{gathered}
\frac{\partial u}{\partial x}+\frac{\partial v}{\partial y}+\frac{\partial w}{\partial z}=0 \\
\frac{\partial u}{\partial t}+U \frac{\partial u}{\partial x}+\frac{d U}{d y} v+\frac{\partial p}{\partial x}=0 \\
\frac{\partial v}{\partial t}+U \frac{\partial v}{\partial x}+\frac{\partial p}{\partial y}=0
\end{gathered}
$$

and

$$
\frac{\partial w}{\partial t}+U \frac{\partial w}{\partial x}+\frac{\partial p}{\partial z}=0
$$

On using the transformation of variables given by $T=t, \xi=x-U t, \eta=y, \zeta=z$ and the Fourier transformations defined with respect to $\xi$ and $\zeta$ as

$$
\breve{v}(\alpha ; \eta ; \gamma ; T)=\int_{-\infty}^{\infty} \int_{-\infty}^{\infty} v(\xi, \eta, \zeta, T) e^{i(\alpha \xi+\gamma \zeta)} d \xi d \zeta,
$$

equations (1) to (4) become

$$
\begin{gathered}
-i(\alpha \breve{u}+\gamma \breve{w})+\frac{\partial \breve{v}}{\partial \eta}+i \alpha T U_{\eta} \breve{v}=0 \\
\frac{\partial \breve{u}}{\partial T}+U_{\eta} \breve{v}-i \alpha \breve{p}=0 \\
\frac{\partial \breve{v}}{\partial T}+\frac{\partial \breve{p}}{\partial \eta}+i \alpha T U_{\eta} \breve{p}=0
\end{gathered}
$$

and

$$
\frac{\partial \breve{w}}{\partial T}-i \gamma \breve{p}=0
$$


respectively, with $U_{\eta}=d U / d \eta$.

The Squire transformation, written as

$$
\begin{gathered}
\alpha \breve{u}+\gamma \breve{w}=\tilde{\alpha} \tilde{u} \\
-\gamma \breve{u}+\alpha \breve{w}=\tilde{\alpha} \tilde{w}
\end{gathered}
$$

where $\tilde{\alpha}=\left(\alpha^{2}+\gamma^{2}\right)^{1 / 2}$, combined with operations on (7) to (9) enables us to obtain the pair of equations (cf. Criminale and Drazin 1990)

$$
\begin{gathered}
\frac{\partial}{\partial T} \Delta \breve{v}=0 \\
\frac{\partial \tilde{w}}{\partial T}=\sin \phi U_{\eta} \breve{v}
\end{gathered}
$$

where

$$
\triangle=\frac{\partial^{2}}{\partial \eta^{2}}+2 i \alpha T U_{\eta} \frac{\partial}{\partial \eta}-\tilde{\alpha}^{2}-\alpha^{2} T^{2} U_{\eta}^{2}
$$

and $\sin \phi=\gamma / \tilde{\alpha}$. It is clear that the solutions of (12) and (13) combined with continuity and the Squire transformation are equivalent to solving (6)-(9). Likewise, $\breve{p}$ can be determined from (9). In either case, solutions of the equations are subject to initial conditions and the restriction of boundedness as $\eta \rightarrow \pm \infty$. It should be noted that the fact that the mean velocity has been assumed as only a linear function of $y$ has been explicitly incorporated into the derivation of (12), (13) and (14).

The prototypical family of free shear flows are depicted in figure 1 . As can be seen, the jet and wake can be combined to form one principal family while the mixing layer stands alone. In each case, the solutions of the governing equations must be solved in regions. Then, at the interfaces where the piecewise linear velocities join, the solutions are required to be matched. As pointed out by Criminale and Drazin (1990), the matching requires both $\breve{v}$ and $\breve{p}$ to be continuous in an inviscid fluid. In terms of $\breve{v}$, the pressure becomes

$$
-\tilde{\alpha}^{2} \breve{p}=\frac{\partial^{2} \breve{v}}{\partial \eta \partial T}+i \alpha T U_{\eta} \frac{\partial \breve{v}}{\partial T}+2 i \alpha U_{\eta} \breve{v} .
$$

If the length scale, $\mathrm{H}$, is used together with the time scale, $\sigma$, then the equations can be non-dimensionalized. Then, both (12) and (13) can be considered non-dimensional. By allowing that $\Delta \breve{v}=\Omega(\eta)$ at time, $\mathrm{T}=0$, then the equation

$$
\frac{\partial^{2} \breve{v}}{\partial \eta^{2}}+2 i \alpha T \frac{U_{\eta}}{\sigma} \frac{\partial \breve{v}}{\partial \eta}-\left(\tilde{\alpha}^{2}+\alpha^{2} T^{2} \frac{U_{\eta}^{2}}{\sigma^{2}}\right) \breve{v}=\Omega(\eta)
$$

must be solved in order to obtain the generic solutions for $\breve{v}$. On using the change of variable

$$
\breve{v}(\eta, T)=V(\eta, T) e^{-i \alpha T \frac{U_{\eta}}{\sigma} \eta}
$$

equation (16) becomes

$$
\frac{\partial^{2} V}{\partial \eta^{2}}-\tilde{\alpha}^{2} V=\Omega(\eta) e^{i \alpha T \frac{U_{\eta}}{\sigma} \eta}
$$

The general solution of (18) combined with (17) is 


$$
\breve{v}=\left[A(T) e^{\tilde{\alpha} \eta}+B(T) e^{-\tilde{\alpha} \eta}\right] e^{-i \alpha T \frac{U_{\eta}}{\sigma} \eta}+v_{p}(\eta, T)
$$

where $v_{p}$ is the particular solution obtained by solving the equation

$$
\triangle \breve{v}=\left.\triangle \breve{v}\right|_{T=0}
$$

The specific cases of the jet-wake and the mixing layer can now be considered.

\section{JET-WAKE}

The mean flow velocity, with $\eta=y / H$, is taken as

$$
U(\eta)= \begin{cases}U_{0} & \eta>1 \\ U_{c}+\sigma \eta & 0<\eta<1 \\ U_{c}-\sigma \eta & -1<\eta<0 \\ U_{0} & \eta<-1\end{cases}
$$

where, for the triangular jet profile, $U_{0}=0, U_{c}=1, \sigma=-1$; for the wake profile, $U_{0}=1$, $U_{c}=1-Q, \sigma=Q$ with $Q$ a measure of the wake deficit. On substituting the above mean flow into equation (19), we see that the general nondimensional solution for either the jet or wake is given by

$$
\breve{v}= \begin{cases}A(T) e^{-\tilde{\alpha}(\eta-1)} & \eta>1 \\ \left(B(T) e^{\tilde{\alpha} \eta}+C(T) e^{-\tilde{\alpha} \eta}\right) e^{-i \alpha T \eta}+v_{1, p}(\eta, T) & 0<\eta<1 \\ \left(D(T) e^{\tilde{\alpha} \eta}+E(T) e^{-\tilde{\alpha} \eta}\right) e^{i \alpha T \eta}+v_{2, p}(\eta, T) & -1<\eta<0 \\ F(T) e^{\tilde{\alpha}(\eta+1)} & \eta<-1\end{cases}
$$

plus the appropriate solution for $\breve{p}$ determined from equation (15). Here, we have taken the particular solution $v_{p}$ to be non-zero inside the jet-wake region $\left(v_{p}=v_{1, p}\right.$ for $0<\eta<1$ and $v_{p}=v_{2, p}$ for $-1<\eta<0$ ) and zero outside. The unknown functions of time $A(T), \cdots, F(T)$ are determined by requiring continuity of $\breve{v}$ and $\breve{p}$ at the interfaces. On making the matching the result is a linear ordinary differential system in time, namely

$$
\dot{\vec{x}}=-\frac{i \alpha}{2 \tilde{\alpha}} R \vec{x}+\frac{1}{2 \tilde{\alpha}} \vec{f}, \quad \vec{x}(0)=\overrightarrow{0}
$$

where

$$
\begin{gathered}
R=\left[\begin{array}{ccc}
1-2 \tilde{\alpha}-e^{-2 \tilde{\alpha}} & e^{-2 \bar{\alpha}} & e^{-2 \tilde{\alpha}} \\
1-2 \tilde{\alpha}-e^{-2 \bar{\alpha}} & e^{-2 \tilde{\alpha}}-2 & e^{-2 \tilde{\alpha}}-2 \\
0 & e^{-2 \tilde{\alpha}} & 1-2 \tilde{\alpha}
\end{array}\right] \\
\vec{x}=\left[\begin{array}{lll}
B & D & E
\end{array}\right]^{T}, \quad \vec{f}=\left[\begin{array}{lll}
f_{1} & f_{2} & f_{3}
\end{array}\right]^{T}
\end{gathered}
$$

and $(\dot{)})=\frac{d()}{d T}$. Once $B, D$, and $E$ have been determined, the other functions can be found by means of the following relations:

$$
\begin{gathered}
A=\left(B e^{\bar{\alpha}}+C e^{-\tilde{\alpha}}\right) e^{-i \alpha T}+v_{1, p}(1, T) \\
C=D+E-B+v_{2, p}(0, T)-v_{1, p}(0, T), \\
F=\left(D e^{-\bar{\alpha}}+E e^{\tilde{\alpha}}\right) e^{-i \alpha T}+v_{2, p}(-1, T) .
\end{gathered}
$$


We note here that the solution to equation (23) is independent of the particular profile under consideration; the choice of a jet or wake is only obvious when the solution is transformed back to physical space.

The homogeneous solution of $(23)(\vec{f}=0)$ is the usual normal mode solution, with the eigenvalues determined from the matrix $R$. This solution will also dominate for large time even when $\vec{f}$ is present because of the exponential behaviour.

The essential points in treating an initial-value problem in flows of this type have been presented by Drazin and Reid (1981). In summary, any arbitrary description at time $T=0$ will be a function of $x, y, z$ (or $\xi, \eta, \zeta$ in the transformed system). On using the Fourier transforms, the dependence is then on the wave numbers $\alpha, \gamma$ and $\eta$. The solutions of the perturbation problem indicates that there will be a sum of discrete eigenmodes and an integral of a continuous eigenspectrum that can be used to represent the prescribed initial function. The solutions obtained by use of the piecewise linear model provides all of this information explicitly.

The number of eigenvalues in the piecewise linear model is essentially proportional to the number of connections used for the basic velocity profile and hence, in the limit of a continuous profile, there would be an infinite number of modes. The qualitative behaviour of the modes is the same as that known from numerical computations using a continuous $U(y)$, particularly the fact that there is an unstable mode for the interval $0<\tilde{\alpha}<\tilde{\alpha}_{c}$. The differences between the modeled and the continuous $U(y)$ are primarily (a) the value of $\tilde{\alpha}_{c}$ for the modeled profile is somewhat less than the neutral equivalent for the continuous $U(y)$; (b) the modeled $U$ has neutral solutions for all $\tilde{\alpha}>\tilde{\alpha}_{c}$ whereas no eigenvalues have been found using the continuous $U$ for $\tilde{\alpha}>\tilde{\alpha}_{c}$ (cf. Betchov and Criminale 1967). The first point is not significant but the second restricts the validity to the range of small values of $\tilde{\alpha}$. But, even here, it is this region where the dynamics has salient interest.

There is maximum exponential growth for a mode in the interval $0<\tilde{\alpha}<\tilde{\alpha}_{c}$. The eigenfunction corresponding to this mode is symmetric in $\eta$. These facts provide the clues needed to manipulate the initial designation. Enhancement requires a symmetric variation in $\eta$ whereas delay suggests an asymmetric variation initially. At the same time it should be noted that, unless a feedback mechanism is added, exponential temporal growth of a symmetric variation can not be prevented altogether but only delayed.

\subsection{SYMMETRIC DISTURBANCES}

For symmetric initial conditions we solve the following equation to obtain the particular solution

$$
\Delta \breve{v}=\Omega_{0}\left[e^{i \beta_{0} \eta}+e^{-i \beta_{0} \eta}\right] .
$$

Prescribing $\triangle \breve{v}$ at $T=0$ is effectively prescribing vorticity. Moreover, symmetric (or asymmetric) $\Delta \breve{v}$ will result in a symmetric (or asymmetric) initial $\breve{v}$. On using (29),

$$
v_{p}=\Omega_{0}\left[a(T) e^{i \beta_{0} \eta}+b(T) e^{-i \beta_{0} \eta}\right]
$$

where

$$
a(T)=\frac{-1}{\left[\beta_{0}+\alpha \frac{U_{\eta}}{\sigma} T\right]^{2}+\tilde{\alpha}^{2}}, \quad b(T)=\frac{-1}{\left[\beta_{0}-\alpha \frac{U_{\eta}}{\sigma} T\right]^{2}+\tilde{\alpha}^{2}} .
$$

Note that $v_{1, p}$ and $v_{2, p}$ in (22) can now be determined by using the conditions $U_{\eta}=\sigma$ for $0<\eta<1$ and $U_{\eta}=-\sigma$ for $0<\eta<1$. 
The system (23) is now solved numerically using a fourth-order Runge Kutta scheme. All calculations were computed in double precision. In each case reported here and in the following subsection, the magnitude of $D$ was always larger than the magnitude of either $B$ or $E$. For this reason only graphs of $|D|$ are given. In figure 2 we plot $|D|$ as a function of time with $\phi=0$, $\beta_{0}=(2 n-1) \pi / 2$ and for various values of $\alpha$. This choice of $\beta_{0}$ ensures that the particular solution vanishes at $\eta= \pm 1$ and is taken as a convenience. The values of $\alpha$ corresponds to the neutral mode, the maximum growth rate mode and its subharmonic from the normal mode analysis. As $n$ is increased from 1 to 10 , we see that the exponential growth is slightly delayed, suggesting that symmetric initial disturbances do not significantly alter the transient behaviour.

\subsection{ASYMMETRIC DISTURBANCES}

For asymmetric initial conditions we solve the equation for the particular solution

$$
\triangle \breve{v}=\Omega_{0}\left[e^{i \beta_{0} \eta}-e^{-i \beta_{0} \eta}\right]
$$

which results in

$$
v_{p}=\Omega_{0}\left[a(T) e^{i \beta_{0} \eta}+b(T) e^{-i \beta_{0} \eta}\right]
$$

where

$$
a(T)=\frac{-1}{\left[\beta_{0}+\alpha \frac{U_{\eta}}{\sigma} T\right]^{2}+\tilde{\alpha}^{2}}, \quad b(T)=\frac{1}{\left[\beta_{0}-\alpha \frac{U_{\eta}}{\sigma} T\right]^{2}+\tilde{\alpha}^{2}} .
$$

Note that $v_{1, p}$ and $v_{2, p}$ in (22) can now be determined by using the conditions $U_{\eta}=\sigma$ for $0<\eta<1$ and $U_{\eta}=-\sigma$ for $0<\eta<1$.

The system (23) is again solved numerically using a fourth-order Runge Kutta scheme. In figure 3 we plot $|D|$ as a function of time with $\phi=0, \beta_{0}=n \pi$ and for various values of $\alpha$. The choice of $\beta_{0}$ ensures that the particular solution vanishes at $\eta= \pm 1$. The values of $\alpha$ corresponds to the neutral mode, the maximum growth rate mode and its subharmonic from the normal mode analysis. Each sub-figure should be compared to the corresponding sub-figure for the symmetric initial disturbance and special attention should be paid to the scales of each graph. As $n$ is increased from 1 to 10, we see that the exponential growth is significantly delayed, suggesting that asymmetric initial disturbances can alter the transient behaviour. The maxima obtained here for early time are due to the 'Orr or venetian blind effect' and is manifested in the choice of initial conditions being periodic in $\eta$. Mathematically, an inspection of the coefficient $b(T)$ in (31) or (34) can be seen to be the origin both here and in the symmetric initial value.

\section{MIXING LAYER}

The mean flow velocity for the mixing layer is taken as

$$
U(\eta)= \begin{cases}1 & \eta>1 \\ \eta & -1<\eta<1 \\ -1 & \eta<-1\end{cases}
$$

and we have set $\sigma=1$ for convenience. Substituting the above mean flow into equation (19), we see that the general nondimensional solution is given by 


$$
\breve{v}= \begin{cases}A(T) e^{-\tilde{\alpha}(\eta-1)} & \eta>1 \\ \left(B(T) e^{\tilde{\alpha} \eta}+C(T) e^{-\tilde{\alpha} \eta}\right) e^{-i \alpha T \eta}+v_{p}(\eta, T) & -1<\eta<1 \\ D(T) e^{\tilde{\alpha}(\eta+1)} & \eta<-1\end{cases}
$$

plus the appropriate solution for $\breve{p}$ determined from equation (15). Here, we have taken the particular solution to be non-zero inside the mixing layer region and zero outside. As in the previous example, the unknown functions of time $A(T), \cdots, D(T)$ are determined by requiring continuity of $\breve{v}$ and $\breve{p}$ at the interfaces. On making the matching the result is a linear ordinary differential system in time, namely

$$
\dot{\vec{x}}=-\frac{i \alpha}{2 \tilde{\alpha}} R \vec{x}+\frac{1}{2 \tilde{\alpha}} \vec{f}, \quad \vec{x}(0)=\overrightarrow{0}
$$

where

$$
\begin{gathered}
R=\left[\begin{array}{cc}
1-2 \tilde{\alpha} & e^{-2 \tilde{\alpha}} \\
-e^{-2 \tilde{\alpha}} & 2 \tilde{\alpha}-1
\end{array}\right] \\
\vec{x}=[B C]^{T}, \quad \vec{f}=\left[\begin{array}{ll}
f_{1} & f_{2}
\end{array}\right]^{T},
\end{gathered}
$$

and $\left(\dot{)}=\frac{d()}{d T}\right.$. Once $B$ and $C$ have been calculated, the other functions can be found by means of the following relations:

$$
\begin{aligned}
& A=\left(B e^{\bar{\alpha}}+C e^{-\bar{\alpha}}\right) e^{-i \alpha T}+v_{p}(1, T), \\
& D=\left(B e^{-\tilde{\alpha}}+C e^{\bar{\alpha}}\right) e^{i \alpha} T+v_{p}(-1, T)
\end{aligned}
$$

The homogeneous solution of (37) is the usual normal mode solution, with the eigenvalues determined from the matrix R. This solution will also dominate for large time when $\vec{f}$ is present. As in the previous example, we consider the two special cases of symmetric and asymmetric initial disturbances. The particular solution is the same form as in the jet-wake case. The system is again solved numerically using a fourth-order Runge Kutta scheme. In each case reported here, the magnitude of $B$ was always larger than the magnitude of $C$. For this reason only graphs of $|B|$ are given. For the symmetric case, the magnitude of $B$ is plotted in figure 4 as a function of time with $\phi=0, \beta_{0}=(2 n-1) \pi / 2$ and for various values of $\alpha$. Likewise, for the asymmetric case, the magnitude of $B$ is plotted in figure 5 as a function of time with $\phi=0, \beta_{0}=n \pi$ and for various values of $\alpha$. The values of $\alpha$ correspond to the neutral mode, the maximum growth rate mode and its subharmonic from the normal mode analysis. In both cases, as $n$ is increased from 1 to 10 , we see that the exponential growth is delayed, suggesting that high wave number initial disturbances (whether it be symmetric or asymmetric) may delay the eventual rollup.

\section{CONTINUOUS PROFILE ANALYSIS}

As stated there is no problem in using the moving coordinate transformation when the basic profile is a continuous function of the transverse variable y. As before, the transformation from the Cartesian to the moving frame is given by

$$
T=t, \quad \xi=x-U(y) t, \quad \eta=y, \quad \zeta=z,
$$

but now both the equations and the operator must be amended. The same two equations for the perturbation quantities can be obtained in exactly the same manner and they become 


$$
\begin{gathered}
\frac{\partial}{\partial T} \Delta \breve{v}+i \tilde{\alpha} \cos \phi U_{\eta \eta} \breve{v}=0 \\
\frac{\partial \tilde{w}}{\partial T}=\sin \phi U_{\eta} \breve{v}
\end{gathered}
$$

with the operator, $\triangle$, now defined as

$$
\triangle=\frac{\partial^{2}}{\partial \eta^{2}}+2 i \tilde{\alpha} \cos \phi T U_{\eta} \frac{\partial}{\partial \eta}-\tilde{\alpha}^{2}-\tilde{\alpha}^{2} \cos ^{2} \phi T^{2} U_{\eta}^{2}+i \tilde{\alpha} \cos \phi T U_{\eta \eta} .
$$

It is clear that the problem is more complex than that of the constant shear basic profile. Solutions are still subject to the initial and boundary conditions.

Equation (43) could be numerically integrated and these results should be compatible with what has been predicted using the piecewise linear profiles. At least, qualitative agreement should be forthcoming. In lieu of actually doing the numerics here, an analytical procedure can be explored. The basis for the method is due to the fact that the major results for the dynamics of the perturbations of free shear layers occurs for small values of the radial wave number, $\tilde{\alpha}$ (or $\alpha$ if the problem is taken as two-dimensional). Thus, a regular perturbation expansion can be made as $\breve{v}=\breve{v}_{0}+\tilde{\alpha} \breve{v}_{1}+\tilde{\alpha}^{2} \breve{v}_{2}+\cdots$. Substitution of this series into equation (43) generates the set of equations

$$
\begin{gathered}
\frac{\partial}{\partial T}\left(\frac{\partial^{2} \breve{v}_{0}}{\partial \eta^{2}}\right)=0 \\
\frac{\partial}{\partial T}\left(\frac{\partial^{2} \breve{v}_{1}}{\partial \eta^{2}}\right)=-i \cos \phi\left\{2 \frac{\partial}{\partial \eta}\left(U_{\eta} \breve{v}_{0}\right)+T \frac{\partial}{\partial T}\left(2 U_{\eta} \frac{\partial \breve{v}_{0}}{\partial \eta}+U_{\eta \eta} \breve{v}_{0}\right)\right\}
\end{gathered}
$$

for the first two orders. The following observations can be made. First, the lowest order equation (46) is independent of any influence from the mean velocity profile and can be solved in a straight forward manner. Then, the $\breve{v}_{0}$ solution will be time-independent unless it is assumed that there is continuous or time dependent driving in equation (46).

On accepting the time-independent solution for $\breve{v}_{0}$, the $O(\tilde{\alpha})$ equation can be reduced to

$$
\frac{\partial}{\partial T}\left(\frac{\partial^{2} \breve{v}_{1}}{\partial \eta^{2}}\right)=-2 i \cos \phi \frac{\partial}{\partial \eta}\left(U_{\eta} \breve{v}_{0}\right)
$$

and, immediately,

$$
\frac{\partial^{2} \breve{v}_{1}}{\partial \eta^{2}}=-2 i \cos \phi T \frac{\partial}{\partial \eta}\left(U_{\eta} \breve{v}_{0}\right)
$$

where $\partial^{2} \breve{v}_{1} / \partial \eta^{2}=0$ at $\mathrm{T}=0$. The full solution for $\breve{v}_{1}$ is

$$
\breve{v}_{1}=V_{0}+B \eta-2 i \cos \phi T \int U_{\eta} \breve{v}_{0} d \eta
$$

where $V_{0}$ and $B$ are constants. But, $B=0$ by the boundary conditions and therefore

$$
\breve{v}_{1}=V_{0}-2 i \cos \phi T \int U_{\eta} \breve{v}_{0} d \eta \text {. }
$$

It is clear from this series that only algebraic terms in $\mathrm{T}$ will be generated regardless of the influence of the mean profile in the successive equations. Thus, the early period motion is purely algebraic. As time increases the series is no longer valid as can be seen from the 
operator (45). The implication is that a new time scale must be invoked in order to have a viable expansion. In fact, on allowing $\tilde{T}=\tilde{\alpha} T$, then the large time behavior can be evaluated.

It can be readily seen that the operator will lose only the $\tilde{\alpha}^{2}$ term in the expansion with the new time scale. Therefore, the new series for $\breve{v}$ is $\breve{v}=\breve{v}_{0}+\tilde{\alpha}^{2} \breve{v}_{1}+\tilde{\alpha}^{4} \breve{v}_{2}+\cdots$ and the lowest order equation is

$$
\frac{\partial}{\partial \tilde{T}} \tilde{\Delta} \breve{v}_{0}+i \cos \phi U_{\eta \eta} \breve{v}_{0}=0
$$

where $\tilde{\Delta}=\Delta+\tilde{\alpha}^{2}$. In effect, (52) is the full equation and it would seem that there is little to be gained by the small $\tilde{\alpha}$ expansion. Interestingly enough, this is not the case and implications from the full equation can be found without actually resorting to the solution.

By considering the transformation

$$
\breve{v}=V(\eta, \tilde{T}) e^{-i \tilde{T} \cos \phi U(\eta)}
$$

the full equation can be rewritten as

$$
\frac{\partial}{\partial \tilde{T}}\left\{\left(\frac{\partial^{2} V}{\partial \eta^{2}}-\tilde{\alpha}^{2} V\right) e^{-i \tilde{T} \cos \phi U(\eta)}\right\}+i \cos \phi U_{\eta \eta} V e^{-i \tilde{T} \cos \phi U(\eta)}=0 .
$$

Then, if (54) is expanded and $V$ is Laplace transformed to remove the time dependence, then

$$
[s-i \tilde{\alpha} \cos \phi U(\eta)]\left[\frac{d^{2} \bar{V}}{d \eta^{2}}-\tilde{\alpha}^{2} \bar{V}\right]+i \cos \phi U_{\eta \eta} \bar{V}=G(\eta)
$$

where $\bar{V}$ is the Laplace transform of $V$ and $G(\eta)$ is an initial value. This equation is another way of expressing the Rayleigh equation that is dominant in inviscid fluid dynamic stability theory. Consequently, a growing exponential solution can be expected to dominate asymptotically in time.

A final remark should be made with regard to the use of the moving coordinate transformation. Heretofore, small wave number expansions have been used to solve the Rayleigh (or Orr-Sommerfeld) equation but a nonuniformity in the spatial variable $y$ must be treated. Here, this is not a problem as has been shown throughout. Instead, it is time that must be scaled and this makes for an added facility in the investigation.

\subsection{NUMERICAL SOLUTION}

In this subsection the numerical solution to the full equation (43) is presented for both the Bickley jet $\left(U(\eta)=\operatorname{sech}^{2} \eta\right)$ and the mixing layer $(U(\eta)=\tanh \eta)$. It is first convenient to introduce the transformation

$$
\breve{v}=V(\eta, T) e^{-i \bar{\alpha} T \cos \phi U(\eta)},
$$

so that the full equation (44) can be rewritten as

$$
\frac{\partial}{\partial T}\left\{\frac{\partial^{2} V}{\partial \eta^{2}}-\tilde{\alpha}^{2} V\right\}-i \tilde{\alpha} \cos \phi U(\eta)\left\{\frac{\partial^{2} V}{\partial \eta^{2}}-\tilde{\alpha}^{2} V\right\}+i \tilde{\alpha} \cos \phi U_{\eta \eta} V=0
$$

Note that $\phi$ can be scaled out by an appropriate transformation in time. This equation is to be solved subject to appropriate initial conditions. Here, we take 


$$
V(\eta, 0)=\cos \left(\beta_{0} \eta\right) e^{-\eta^{2}}
$$

for symmetric disturbances, or

$$
V(\eta, 0)=\sin \left(\beta_{0} \eta\right) e^{-\eta^{2}}
$$

for asymmetric disturbances, where $\beta_{0}$ is some wave number.

The partial differential equation (57) was solved numerically by the method of lines. The spatial derivatives were center differenced and the resulting system was integrated in time by a Runge Kutta scheme. All calculations were done in double precision. The results were checked for convergence by increasing the number of mesh points and varying the region in $\eta$ over which the differencing was done.

Results for the Bickley jet are shown in figures 6 and 7 . In the top portion of each figure the maximum of the magnitudes of the real and imaginary part of $V$ (denoted by the solid and dashed curves, respectively) are plotted as a function of time. In the bottom portion of each figure the real and imaginary parts of $V$ (denoted by the solid and dashed curves, respectively) are plotted as a function of $\eta$ at the last integrated time step shown in the corresponding top portion. The results shown in figure 6 correspond to the case of the symmetric initial disturbance (58), and the results shown in figure 7 correspond to asymmetric initial disturbances (59). In each case we have taken $\beta_{0}=1, \phi=0$ and $\tilde{\alpha}=1$. Note that these results are qualitatively similar for those of the piecewise linear profiles shown in figures 2 and 3, respectively. We are therefore lead to the conclusion that an asymmetric initial disturbance can significantly delay the eventual exponential growth, and that this feature is insensitive to either the actual jet profile or the form of the initial disturbance taken. Although not done here, similar conclusions can be expected for the wake.

Results for the mixing layer are shown in figures 8 through 10 . The nomenclature is the same as in the previous set of figures. The results shown in figure 8 correspond to the case of the symmetric initial disturbance (58), and the results shown in figure 9 correspond to asymmetric initial disturbances (59). In each case we have taken $\beta_{0}=1, \phi=0$ and $\tilde{\alpha}=0.396$. For the $\beta_{0} \approx O(1)$ wave number values, the time at which exponential growth becomes evident are similar. Figure 10 shows results for the asymmetric initial disturbance but at a higher wave number, namely, $\beta_{0}=10 \pi$. Note that the higher wave number delays somewhat the eventual exponential growth, thereby delaying the rollup of the vortices. These results are qualitatively similar to those for the piecewise linear profile shown in figures 4 and 5 , respectively. We therefore are lead to the conclusions that symmetric or asymmetric initial disturbances with $O(1)$ wave numbers do not significantly alter the eventual exponential growth while for larger

wave numbers the exponential growth is delayed, and that these features are insensitive to either the actual mixing layer profile or the form of the initial disturbance taken.

\section{EFFECT OF THREE-DIMENSIONALITY}

The perturbation problem is complete when all components of the fluctuation velocity and the pressure are known. As noted, by using the Squire mode equation (13) for $\tilde{w}$ together with the dominant equation (12) for $\breve{v}$, this task is accomplished when the solutions of these two equations are obtained. In this way, not only can all disturbance quantities be derived from these two variables, but the effects of obliquity in the field are ascertained. It is not sufficient to use the Squire's theorem from stability analysis since this theorem is only valid for separable 
normal modes. The presence of the continuous spectrum in the initial-value calculations requires further scrutiny.

Some effects due to the Squire theorem are, of course, present in the representation. This can be seen directly by examining the equation for $\breve{v}$. Except for the angle $\phi=\pi / 2$, the implications of obliquity can be inferred. Note that, in the equation for $\breve{v}$, if $T$ is replaced by the new time $T^{\prime}=T \cos \phi$, then $\phi$ no longer appears. Thus, any angle where $0<\phi<\pi / 2$, the temporal behavior of $\breve{v}$ mirrors that of $\phi=0(\gamma=0)$ except that it responds more slowly and with a decreased amplitude, depending on the value of $\cos \phi$. The role of the other velocity components $\breve{u}, \breve{w}$ (or $\tilde{u}, \tilde{w}$ ) must, however, be viewed with some caution since these quantities retain an explicit dependence on $\phi$.

Unlike the governing equation for $\breve{v}$, the one for $\tilde{w}$ cannot suppress the angle $\phi$. On the other hand, this equation can be integrated at once to give

$$
\tilde{w}=W_{0}(\eta)+\sin \phi U_{\eta} \int_{0}^{T} \breve{v}\left(\eta, T^{\prime}\right) d T^{\prime}
$$

where $W_{0}(\eta)=\tilde{w}(\eta, 0)$. In general it can be concluded that any exponential dependence on $T$ from $\breve{v}$ will remain exponential for $\tilde{w}$ whereas algebraic dependence on $T$ will result in a raising of the power of $T$.

When $\phi=\pi / 2$, then it can be shown that $\breve{v}$ no longer is a function of $T$ (cf. Ellingsen and Palm 1975) and can be written as $\breve{v}=\breve{v}_{0}(\eta)$. The solution for $\tilde{w}$ for this case is simply

$$
\tilde{w}=W_{0}(\eta)+T U_{\eta} \breve{v}_{0}(\eta)
$$

indicating that only a linear algebraic variation is possible.

On returning to the expressions for the Squire transformation (10) and (11), inverting and using continuity for $\tilde{u}$, then

$$
\begin{aligned}
& \breve{u}=-i \cos \phi\left[\frac{1}{\tilde{\alpha}} \frac{\partial \breve{v}}{\partial \eta}+i \cos \phi T U_{\eta} \breve{v}\right]-\sin \phi \tilde{w} \\
& \breve{w}=-i \sin \phi\left[\frac{1}{\tilde{\alpha}} \frac{\partial \breve{v}}{\partial \eta}+i \cos \phi T U_{\eta} \breve{v}\right]+\cos \phi \tilde{w}
\end{aligned}
$$

is found for the velocity components that are in the plane perpendicular to $\breve{v}$. The fact that the solutions for $\breve{v}$ are proportional to the factor $e^{-i \alpha T U}$ as given by (56) reduces the above relations to

$$
\breve{u}=-\frac{i \cos \phi}{\tilde{\alpha}} \frac{\partial V}{\partial \eta} e^{-i \alpha T U}-\sin \phi \tilde{w}
$$

and

$$
\breve{w}=-\frac{i \sin \phi}{\tilde{\alpha}} \frac{\partial V}{\partial \eta} e^{-i \alpha T U}+\cos \phi \tilde{w}
$$

The extreme limits of $\phi=0$ and $\phi=\pi / 2$ contain interesting information. First, for $\phi=0$,

$$
\begin{gathered}
\breve{u}=-\frac{i}{\alpha} \frac{\partial V}{\partial \eta} e^{-i \alpha T U} \\
\breve{w}=\tilde{w}=W(\eta)
\end{gathered}
$$

and the temporal behavior is effectively the same as that determined for $\breve{v}$ in that only the phase variation is involved and not the amplitude. 
When $\phi=\pi / 2$, the limits are

$$
\begin{gathered}
\breve{u}=-\tilde{w}=-\left(W(\eta)+T U_{\eta} \breve{v}_{0}(\eta)\right) \\
\breve{w}=-\frac{i}{\gamma} \frac{\partial V}{\partial \eta}=-\frac{i}{\gamma} \frac{\partial \breve{v}_{0}}{\partial \eta} .
\end{gathered}
$$

Here, the results are quite different, namely (a) only a simple algebraic variation is present and that is only for one of the velocity components; (b) the rate for the linear increase in $\breve{u}$ depends upon the location in $y$ since the slope for the $T$ dependence is the function $\breve{v}_{0}(\eta)$. As has been noted by others, this kind of behavior augurs for formation of streaks and can be expected to be quite predominant at the locations where $\breve{v}_{0}(\eta)$ has its largest values.

The overall effect of any disturbance will depend more realistically on a variety as well as many wave numbers, reflecting the initial distribution. The infinite wave train used in the assumption that the vorticity was periodic in $\mathrm{x}$ and $\mathrm{z}$ used here is but a simplistic limit of more rational distributions, such as finite wave packets, for example. Nevertheless the dynamics as outlined will control the subsequent development.

\section{CONCLUSIONS}

Free shear flows in an incompressible inviscid fluid, consisting of the prototypical jet, wake, and the mixing layer, have been investigated subject to the influence of small perturbations. Instead of using the techniques of classical stability analysis for this purpose, the approach has both posed and solved the complete initial-value problem. Consequently, the full range of the dynamics, including the early transient and the asymptotic fate of any disturbance becomes known. In turn, this information provides a basis by which the flow can be manipulated and the possibility of enhancement or delay of the perturbation field can be considered.

The initial-value problem has been considered heretofore but not generally with the premise of requiring full details. The principal reasons for this lack is that of the mathematics; the physical problem is well posed. Specifically: (a) the governing equations are not of the selfadjoint variety and (b) both a discrete and a continuous spectrum of eigenvalues must be known in order to make any arbitrary initial prescription. Otherwise an expansion would be severely limited. Thus, classical normal mode solutions are not useful here since only the discrete spectrum results. Use of Laplace transforms (cf. Gustavsson 1979) will, in principle, enable this task to be accomplished but the necessary inversions in order to find the temporal behavior are formidable.

This work has as its starting premise the use of moving coordinates. Then, by knowing that classical theory has shown that there are exponentially growing solutions (and therefore unstable) for small values of the wave numbers, a modeled mean flow is taken by using piecewise linear variations for the velocity together with an expansion scheme using continuous profiles. The results indicate that the early time behavior is always algebraic (and due to the continuous spectrum) and the asymptotic fate follows that due to an exponentially growing normal mode (discrete spectrum). Closed form solutions can be obtained as functions of all space variables and time. Effects due to three-dimensionality can also be addressed.

The benefits of the moving coordinate transformation not only demonstrates the type of variation for both early and long times but has the additional benefits that (1) solutions can be found that do not have spatial nonuniformity, and (2) there are no critical layers. Instead, it becomes a two-time problem. The results of the calculations indicate that perturbations in the 
jet-wake family can be delayed to a considerable degree but not altogether eliminated without a feedback mechanism. At the same time, any delay in the mixing layer is modest indeed. In the other extreme, trying to enhance the mixing layer dynamics is likewise futile unless one uses perturbations of finite amplitude. The explanation seems to rests with the basic flows per se. The jet-wake can be visualized as a pair of counter-rotating vortices, one above and one below the center line with no vertical velocity on the center line. A perturbation velocity in this direction that is nonzero at this location (symmetric $v$ ) tends to destroy this configuration as time goes on and the two vortices merge to form one downstream. Naturally, an asymmetric $v$ has an opposite effect and the state with two vortices is prolonged. The mixing layer picture is that of only one vortex and therefore has a nonzero vertical velocity at the center line at the outset. For this reason, an asymmetric $v$ is not at all influential and one that is symmetric can only enhance the process if it is of large enough amplitude and at the correct frequency corresponding to unstable modes.

Finally, direct numerical integration of the partial differential equation derived by use of the moving coordinates was done and it was demonstrated that all of the predictions made by the piecewise linear basic profiles are qualitatively correct. It is suggested that the use of this novel equation may well benefit exploration of other shear flows particularly when attention is directed towards initial value problems. Moreover, it is this equation that can be the basis when contemplating flow control or other salient mechanisms such as receptivity. 


\section{References}

Betchov, R. and Criminale, W.O. (1967). Stability of Parallel Flows, Academic Press.

Bun, Y. and Criminale, W.O. (1994). "Early Period Dynamics of an Incompressible Mixing Layer", J. Fluid Mech., in press.

Criminale, W.O. and Drazin, P.G. (1990). "The evolution of linearized perturbations of parallel flows", Stud. Appl. Math., 83, 123-157.

Drazin, P.G. and Reid, W.H. (1981). Hydrodynamic Stability, Cambridge University Press.

Ellingsen, T. and Palm, E. (1975). "Stability of linear flow", Phys. Fluids, 18, 487-488.

Gustavsson, L.H. (1979). "Initial-value problem for boundary layer flows", Phys. Fluids, 22, 1602-1605.

Kelvin, W. (1887). Philos. Mag., 24, 188-196.

Orr, W. M'F. (1907a). "The stability or instability of the steady motions of a perfect liquid and a viscous liquid. Part I.", Proc. Roy. Irish. Acad., 27, 9-68.

Orr, W. M'F. (1907b). "The stability or instability of the steady motions of a perfect liquid and a viscous liquid. Part II.", Proc. Roy. Irish. Acad., 27, 69-138. 


\section{FIGURE CAPTIONS}

Figure 1. Schematic of family of free shear flows.

Figure 2. Plot of $|D|$ versus time $T$ for symmetric initial disturbance with $\phi=0, \beta_{0}=$ $(2 n-1) \pi / 2$ and (A) $\alpha=1.832744$ (neutral mode), (B) $\alpha=1.22$ (maximum), and (C) $\alpha=0.61$ (subharmonic).

Figure 3. Plot of $|D|$ versus time $T$ for asymmetric initial disturbance with $\phi=0, \beta_{0}=n \pi / 2$ and (A) $\alpha=1.832744$ (neutral mode), (B) $\alpha=1.22$ (maximum), and (C) $\alpha=0.61$ (subharmonic).

Figure 4. Plot of $|B|$ versus time $T$ for symmetric initial disturbance with $\phi=0, \beta_{0}=$ $(2 n-1) \pi / 2$ and (A) $\alpha=0.639232$ (neutral mode), (B) $\alpha=0.396$ (maximum), and (C) $\alpha=0.198$ (subharmonic).

Figure 5. Plot of $|B|$ versus time $T$ for asymmetric initial disturbance with $\phi=0, \beta_{0}=n \pi / 2$ and (A) $\alpha=0.639232$ (neutral mode), (B) $\alpha=0.396$ (maximum), and (C) $\alpha=0.198$ (subharmonic).

Figure 6. (A) Plot of the maximum magnitudes of real (solid) and imaginary (dashed) parts of $V$ as a function of time $T$ for symmetric initial disturbance for $\phi=0, \alpha=1$ and $\beta_{0}=1$. (B) Plot of real (solid) and imaginary (dashed) parts of $V$ as a function of $\eta$ at $T=20$ for $\phi=0$ and $\beta_{0}=1$.

Figure 7. (A) Plot of the maximum magnitudes of real (solid) and imaginary (dashed) parts of $V$ as a function of time $T$ for asymmetric initial disturbance for $\phi=0, \alpha=1$ and $\beta_{0}=1$. (B) Plot of real (solid) and imaginary (dashed) parts of $V$ as a function of $\eta$ at $T=198$ for $\phi=0$ and $\beta_{0}=1$.

Figure 8. (A) Plot of the maximum magnitudes of real (solid) and imaginary (dashed) parts of $V$ as a function of time $T$ for symmetric initial disturbance for $\phi=0, \alpha=0.396$ and $\beta_{0}=1$. (B) Plot of real (solid) and imaginary (dashed) parts of $V$ as a function of $\eta$ at $T=17.6$ for $\phi=0$ and $\beta_{0}=1$.

Figure 9. (A) Plot of the maximum magnitudes of real (solid) and imaginary (dashed) parts of $V$ as a function of time $T$ for asymmetric initial disturbance for $\phi=0, \alpha=0.396$ and $\beta_{0}=1$. (B) Plot of real (solid) and imaginary (dashed) parts of $V$ as a function of $\eta$ at $T=15.4$ for $\phi=0$ and $\beta_{0}=1$.

Figure 10. (A) Plot of the maximum magnitudes of real (solid) and imaginary (dashed) parts of $V$ as a function of time $T$ for asymmetric initial disturbance for $\phi=0, \alpha=0.396$ and $\beta_{0}=1$. (B) Plot of real (solid) and imaginary (dashed) parts of $V$ as a function of $\eta$ at $T=58$ for $\phi=0$ and $\beta_{0}=10 \pi$. 


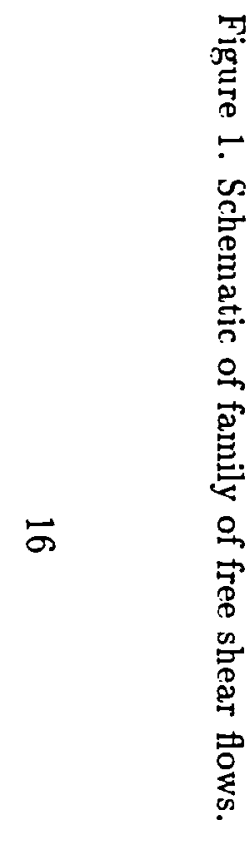

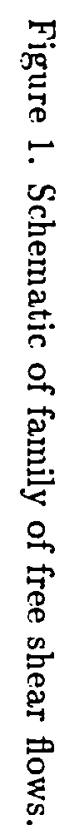

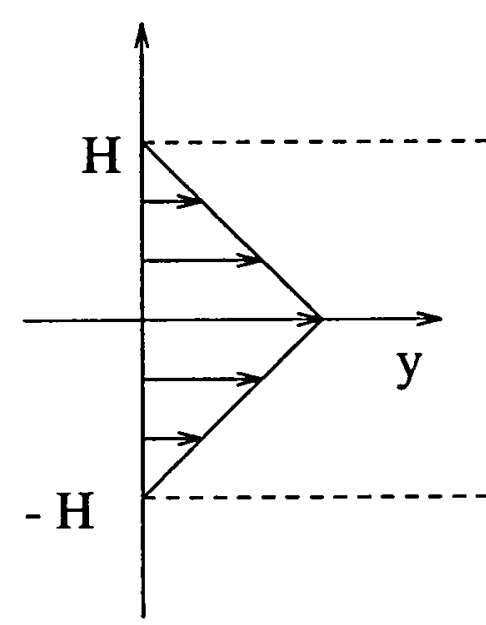

JET

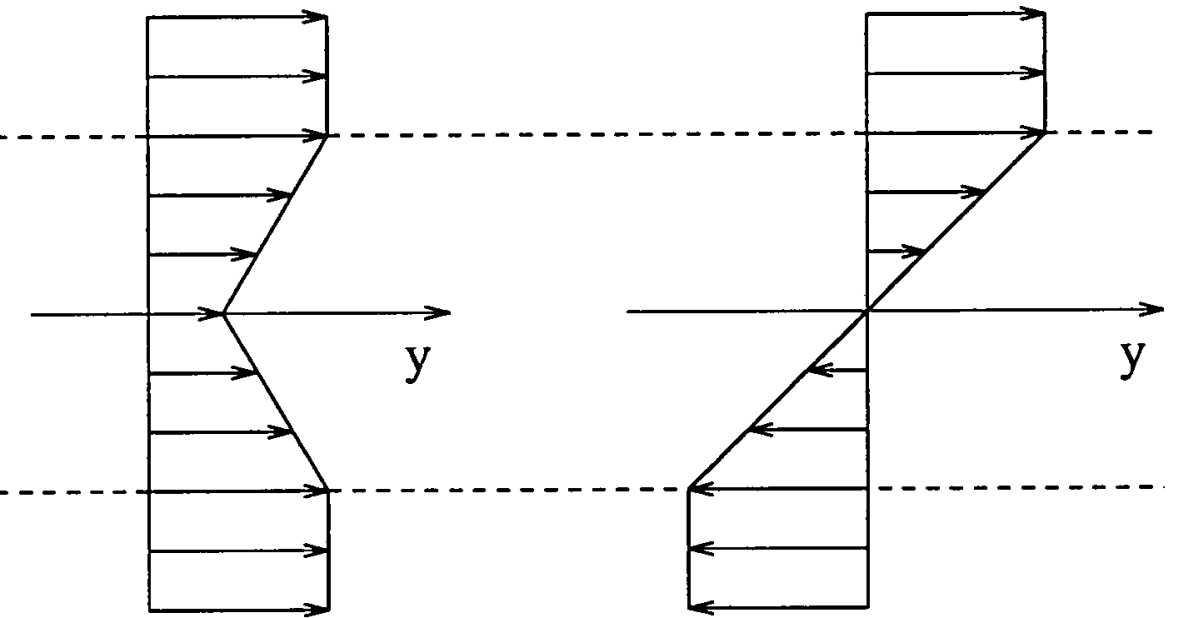

WAKE MIXING LAYER 


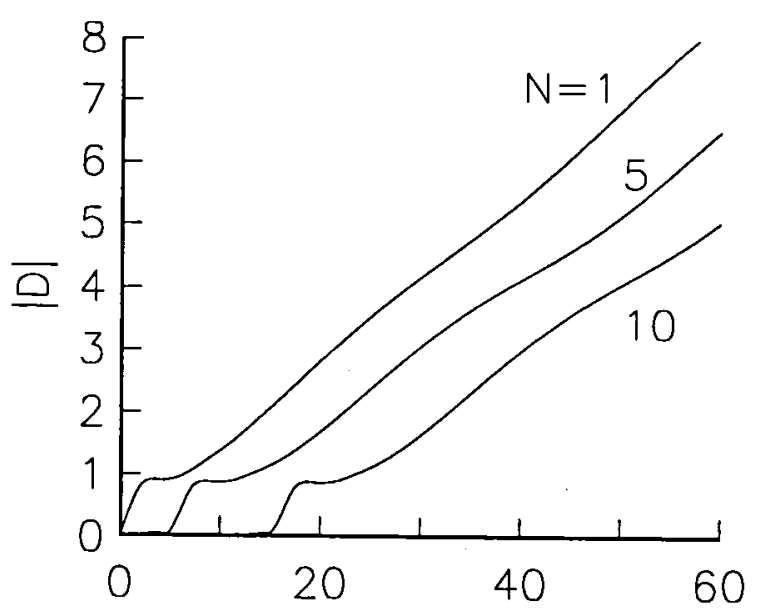

(A)

(B)
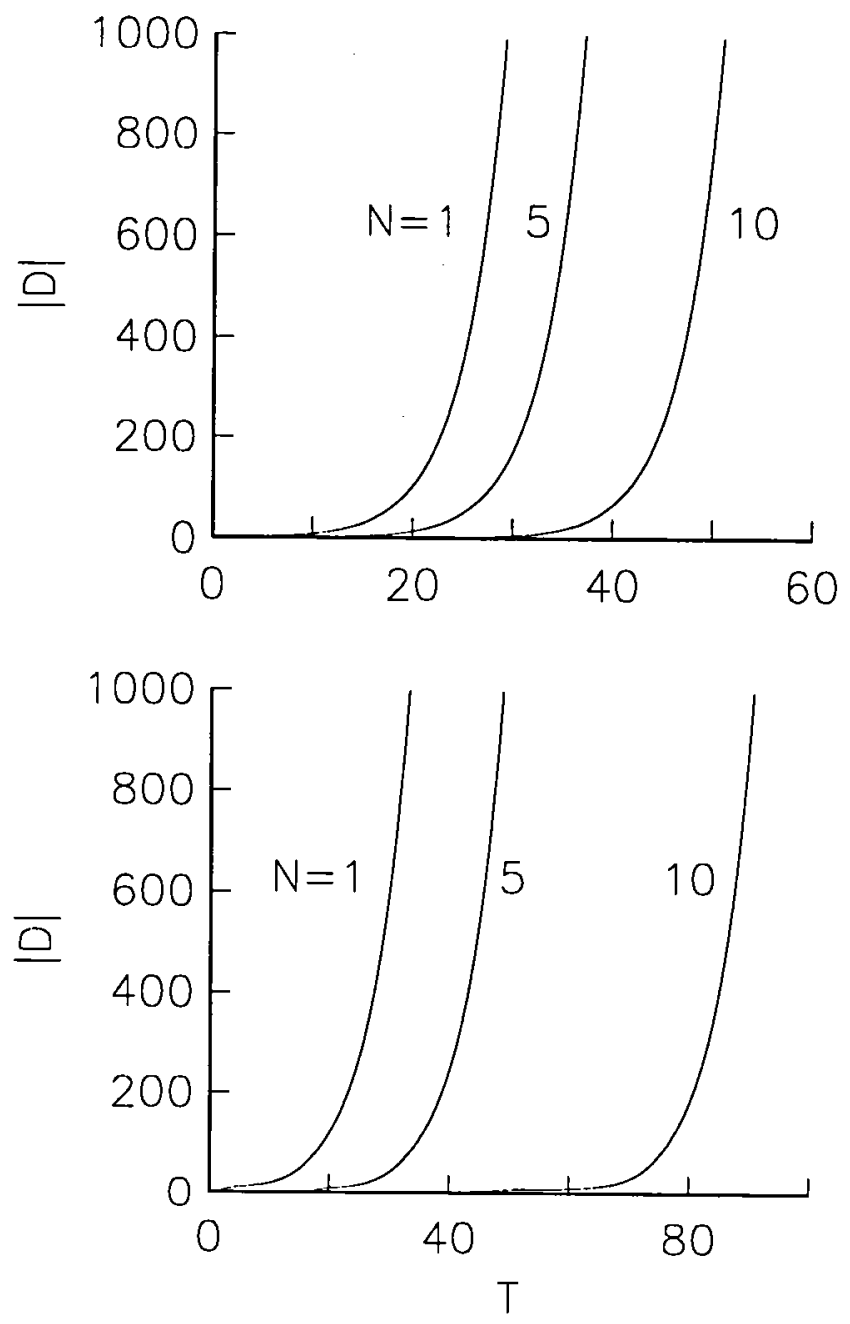

(C)

Figure 2. Plot of $|D|$ versus time $T$ for symmetric initial disturbance with $\phi=0, \beta_{0}=(2 n-1) \pi / 2$ and (A) $\alpha=1.832744$ (neutral mode), (B) $\alpha=1.22$ (maximum), and (C) $\alpha=0.61$ (subharmonic). 


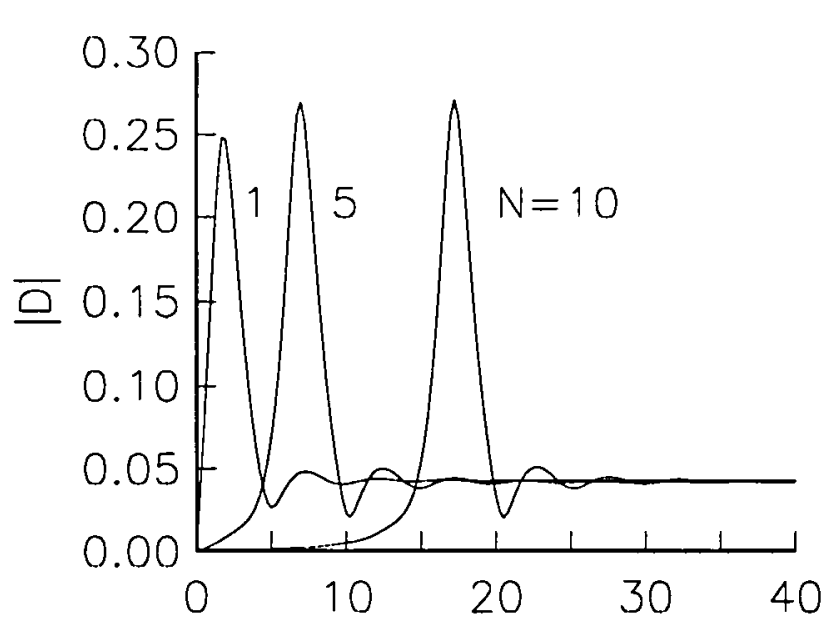

(A)

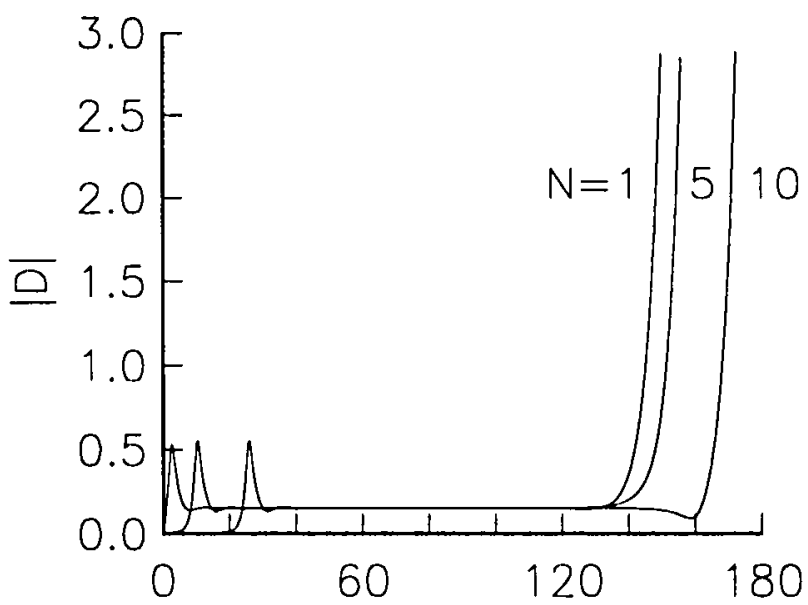

(B)

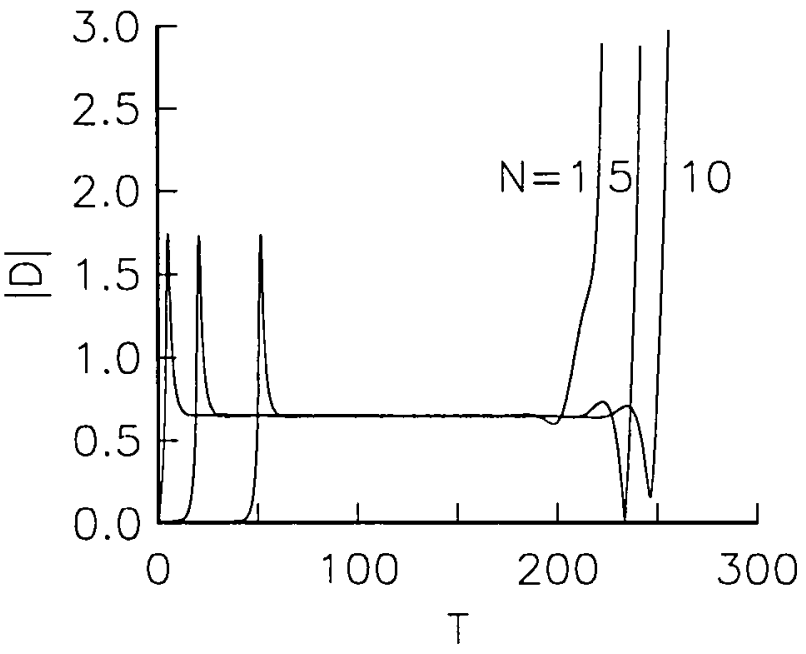

(C)

Figure 3. Plot of $|D|$ versus time $T$ for asymmetric initial disturbance with $\phi=0, \beta_{0}=n \pi / 2$ and (A) $\alpha=1.832744$ (neutral mode), (B) $\alpha=1.22$ (maximum), and (C) $\alpha=0.61$ (subharmonic). 


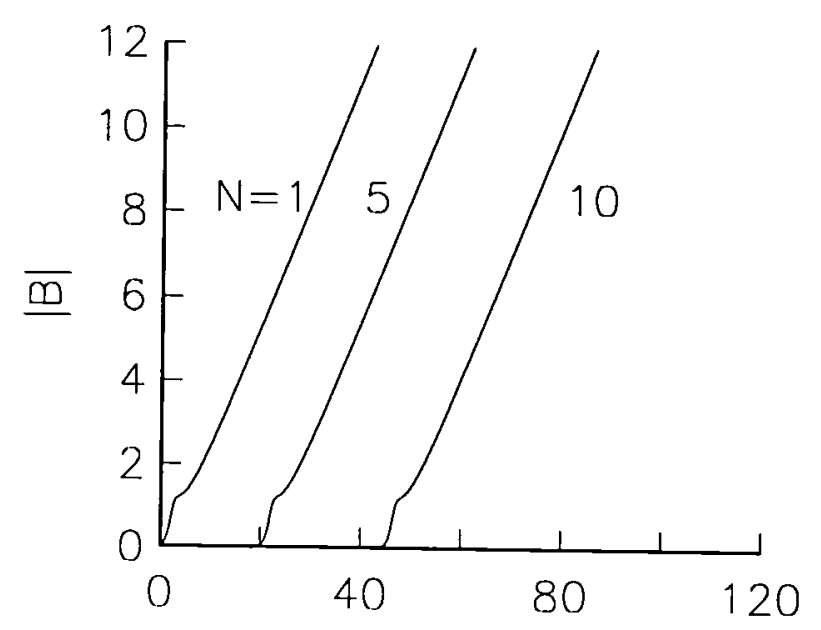

(A)

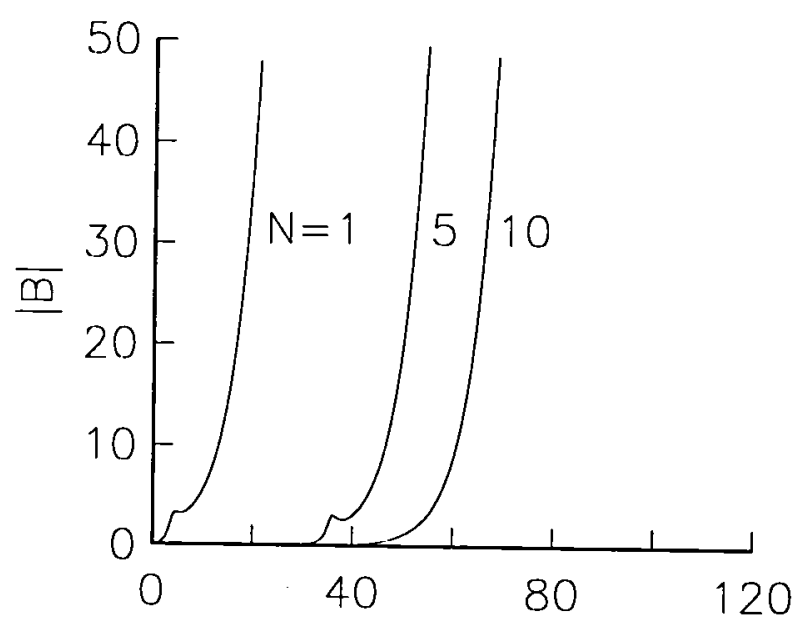

(B)

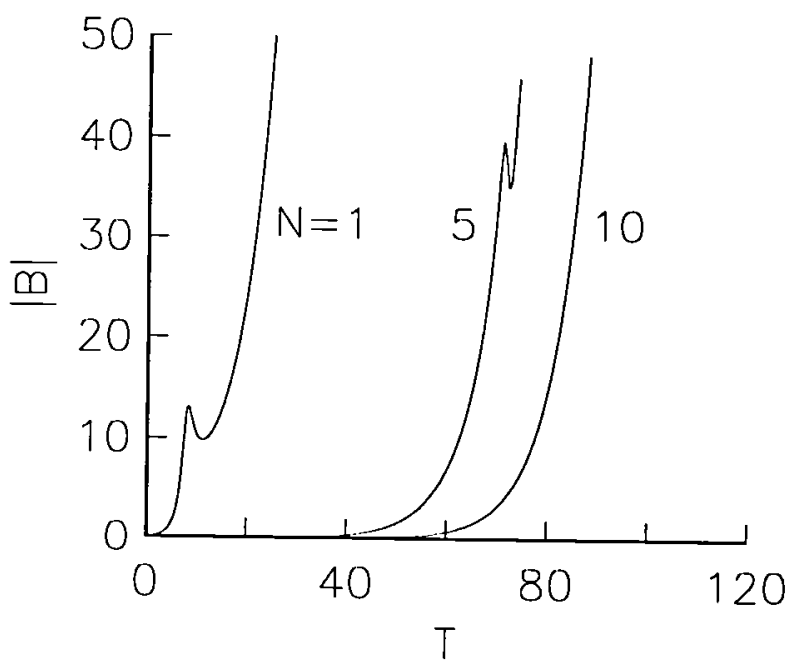

(C)

Figure 4. Plot of $|B|$ versus time $T$ for symmetric initial disturbance with $\phi=0, \beta_{0}=(2 n-1) \pi / 2$ and $(\mathrm{A}) \alpha=0.639232$ (neutral mode), (B) $\alpha=0.396$ (maximum), and (C) $\alpha=0.198$ (subharmonic). 


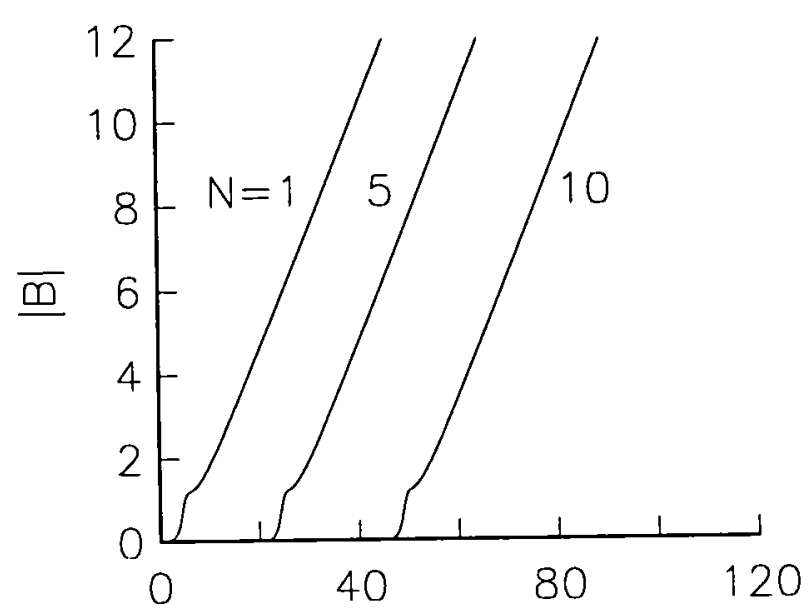

(A)

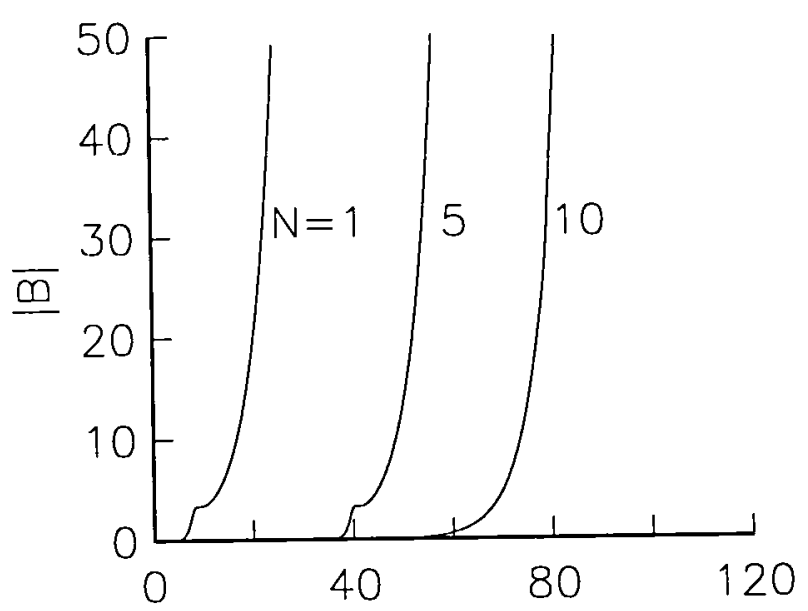

(B)

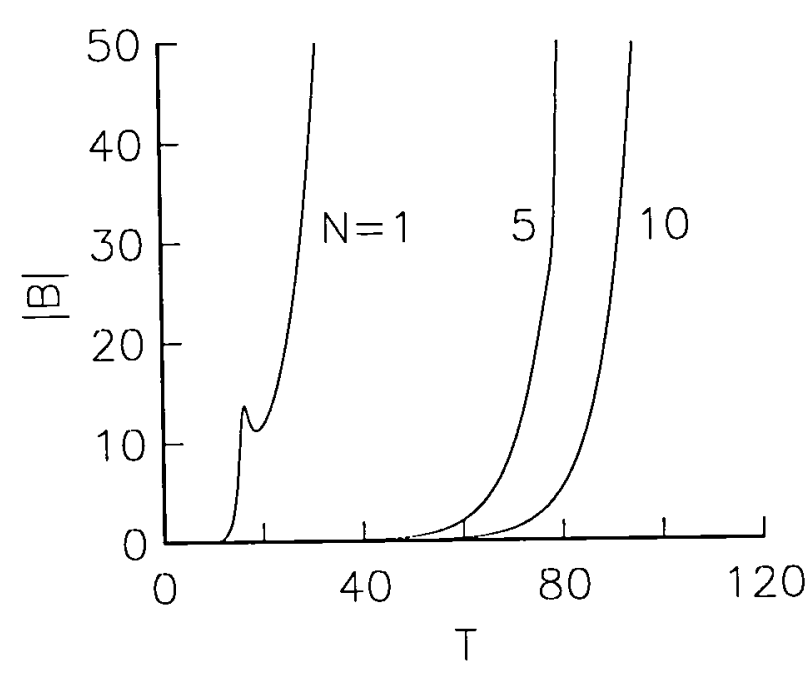

(C)

Figure 5. Plot of $|B|$ versus time $T$ for asymmetric initial disturbance with $\phi=0, \beta_{0}=n \pi / 2$ and $(\mathrm{A}) \alpha=0.639232$ (neutral mode), (B) $\alpha=0.396$ (maximum), and (C) $\alpha=0.198$ (subharmonic). 


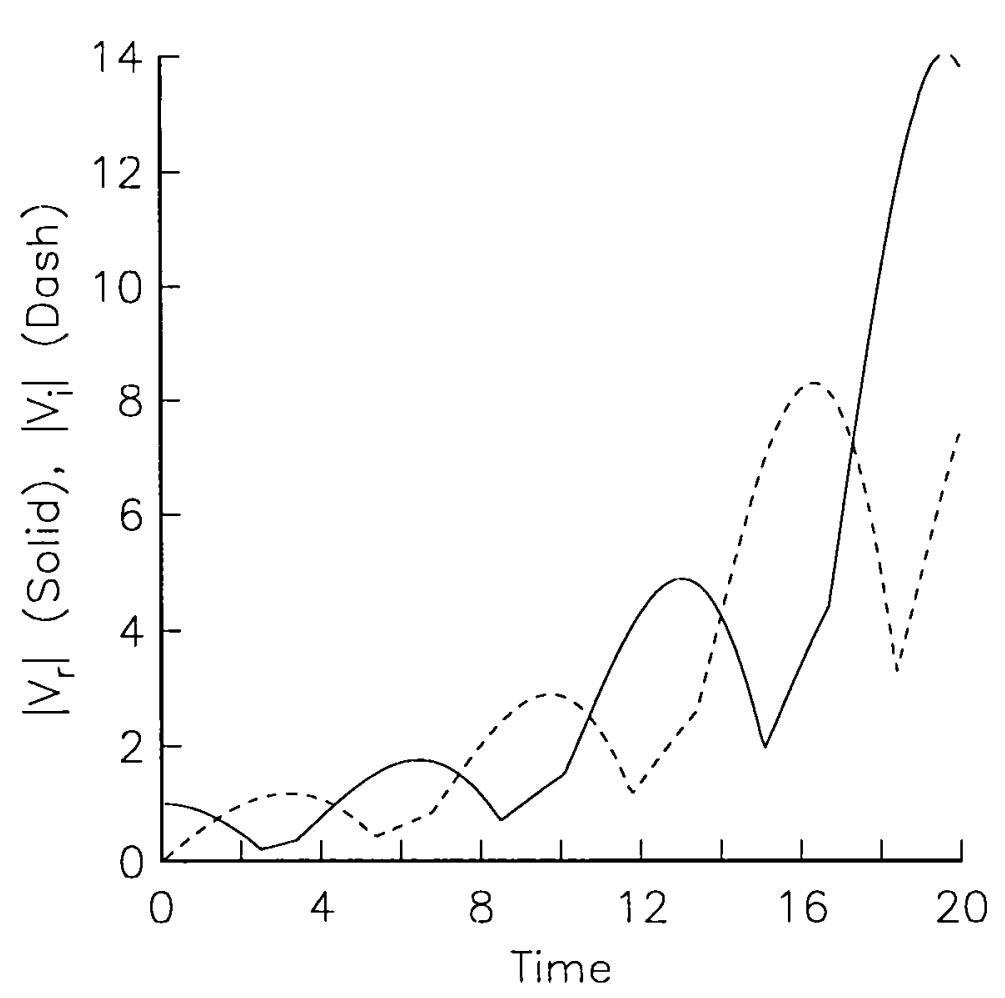

(A)

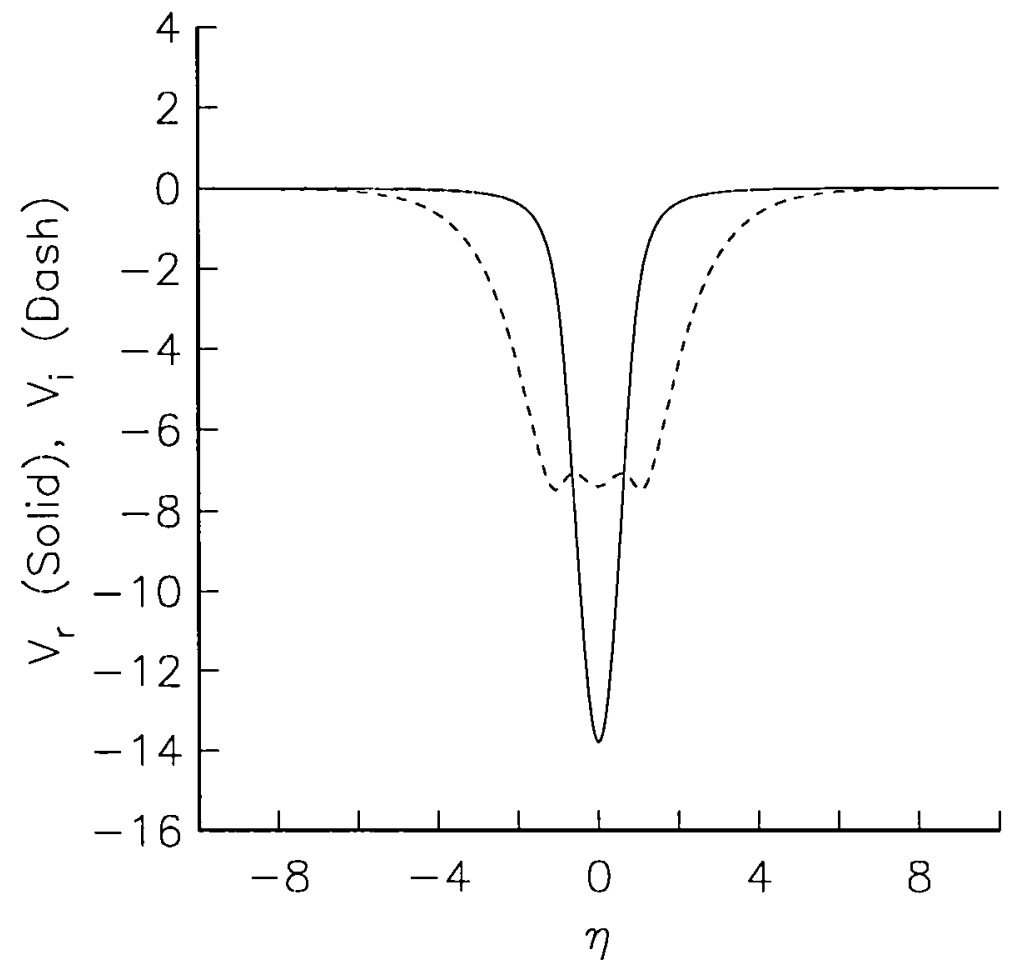

(B)

Figure 6. (A) Plot of the maximum magnitudes of real (solid) and imaginary (dashed) parts of $V$ as a function of time $T$ for symmetric initial disturbance for $\phi=0, \alpha=1$ and $\beta_{0}=1$. (B) Plot of real (solid) and imaginary (dashed) parts of $V$ as a function of $\eta$ at $T=20$ for $\phi=0$ and $\beta_{0}=1$. 


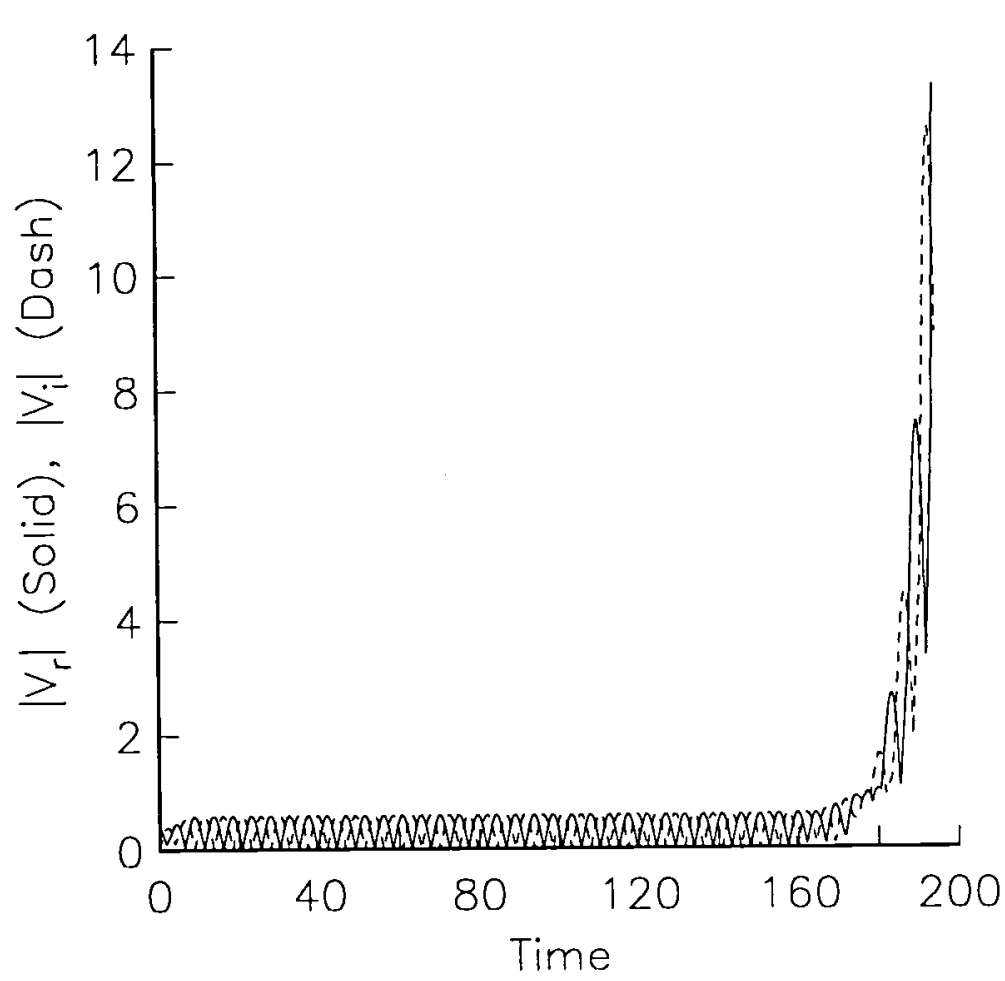

(A)

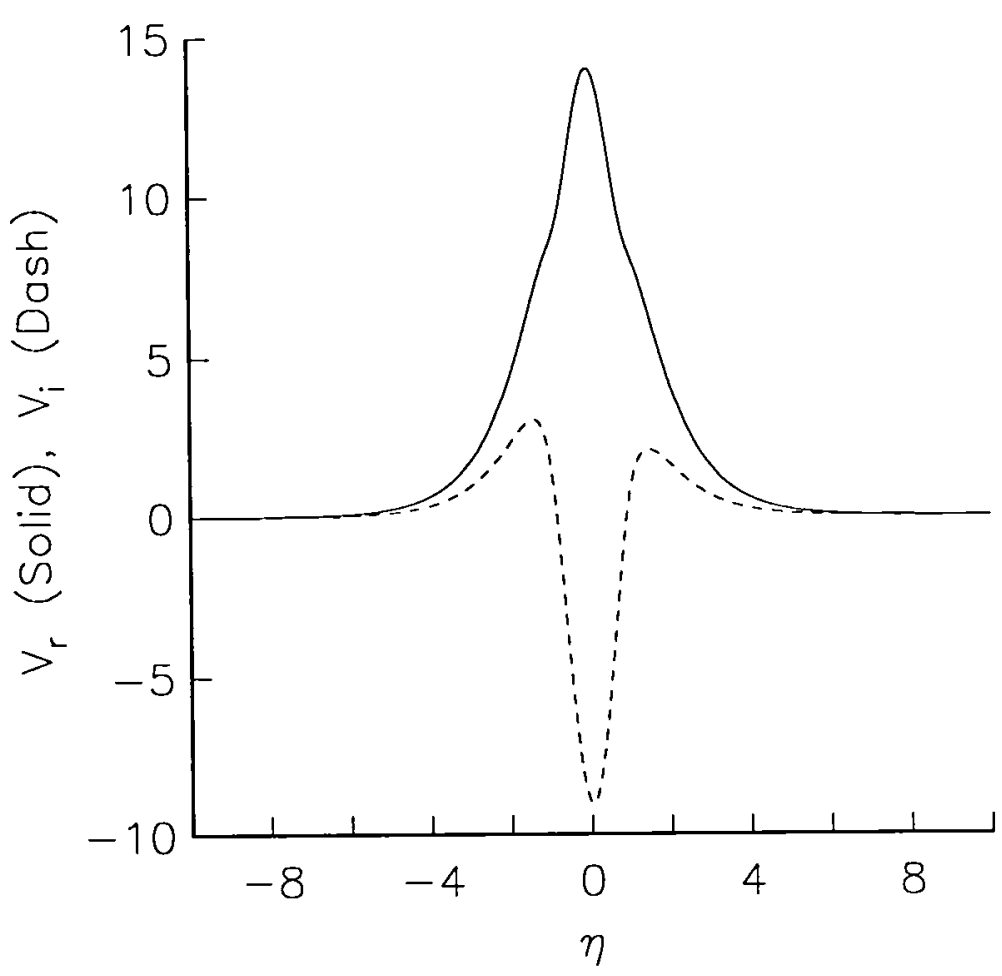

(B)

Figure 7. (A) Plot of the maximum magnitudes of real (solid) and imaginary (dashed) parts of $V$ as a function of time $T$ for asymmetric initial disturbance for $\phi=0, \alpha=1$ and $\beta_{0}=1$. (B) Plot of real (solid) and imaginary (dashed) parts of $V$ as a function of $\eta$ at $T=198$ for $\phi=0$ and $\beta_{0}=1$. 


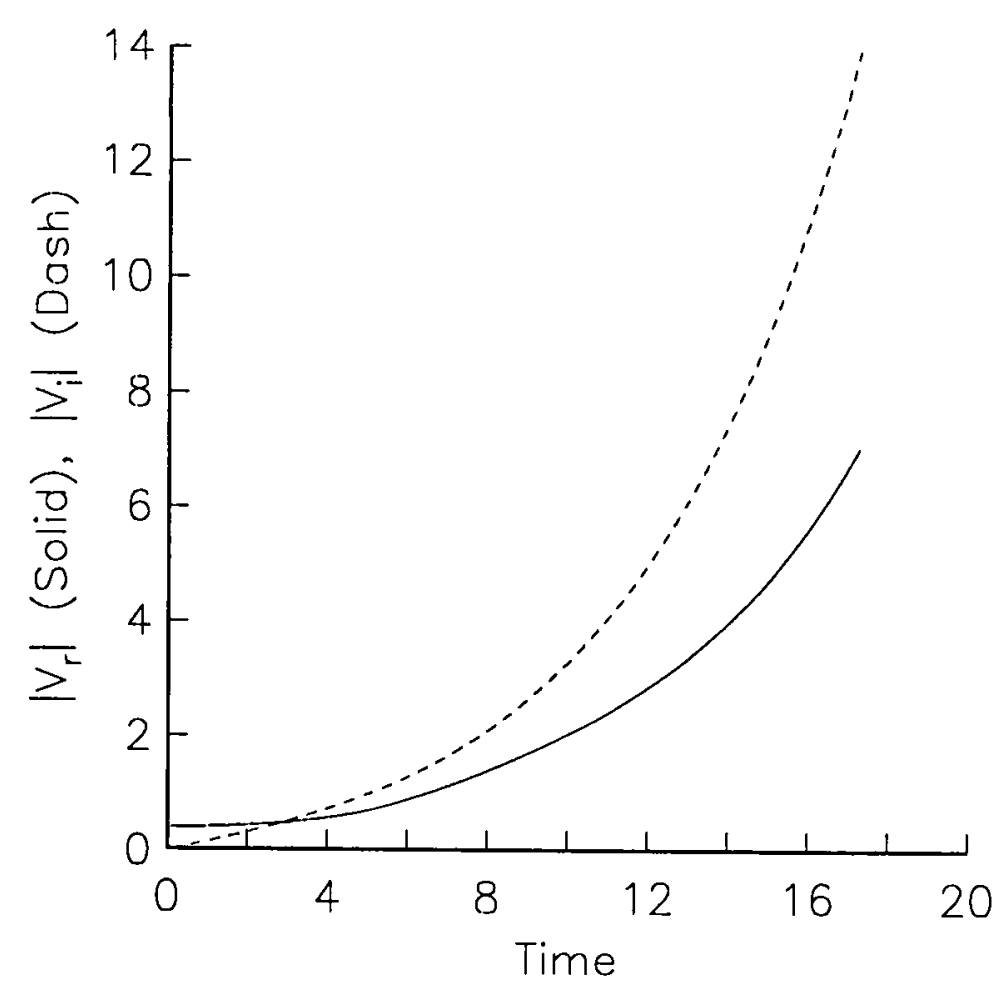

(A)

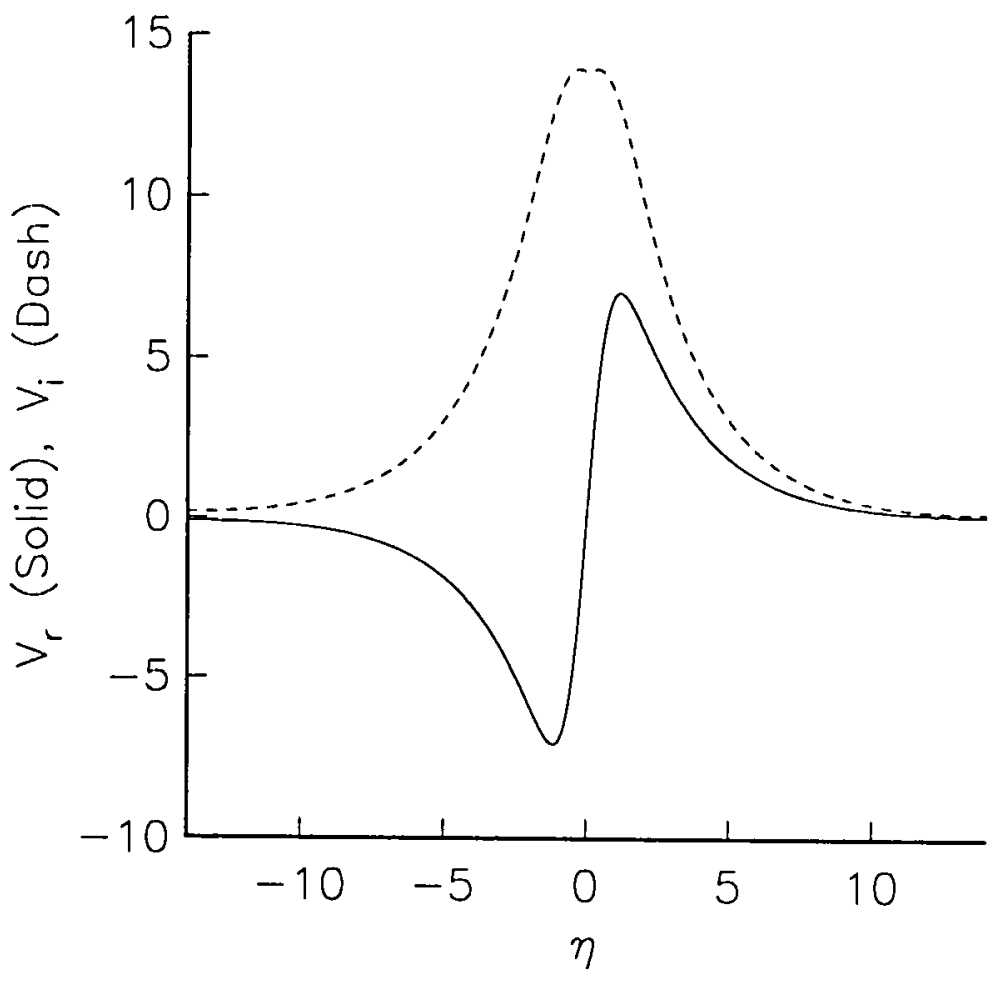

(B)

Figure 8. (A) Plot of the maximum magnitudes of real (solid) and imaginary (dashed) parts of $V$ as a function of time $T$ for symmetric initial disturbance for $\phi=0, \alpha=0.396$ and $\beta_{0}=1$. (B) Plot of real (solid) and imaginary (dashed) parts of $V$ as a function of $\eta$ at $T=17.6$ for $\phi=0$ and $\beta_{0}=1$. 


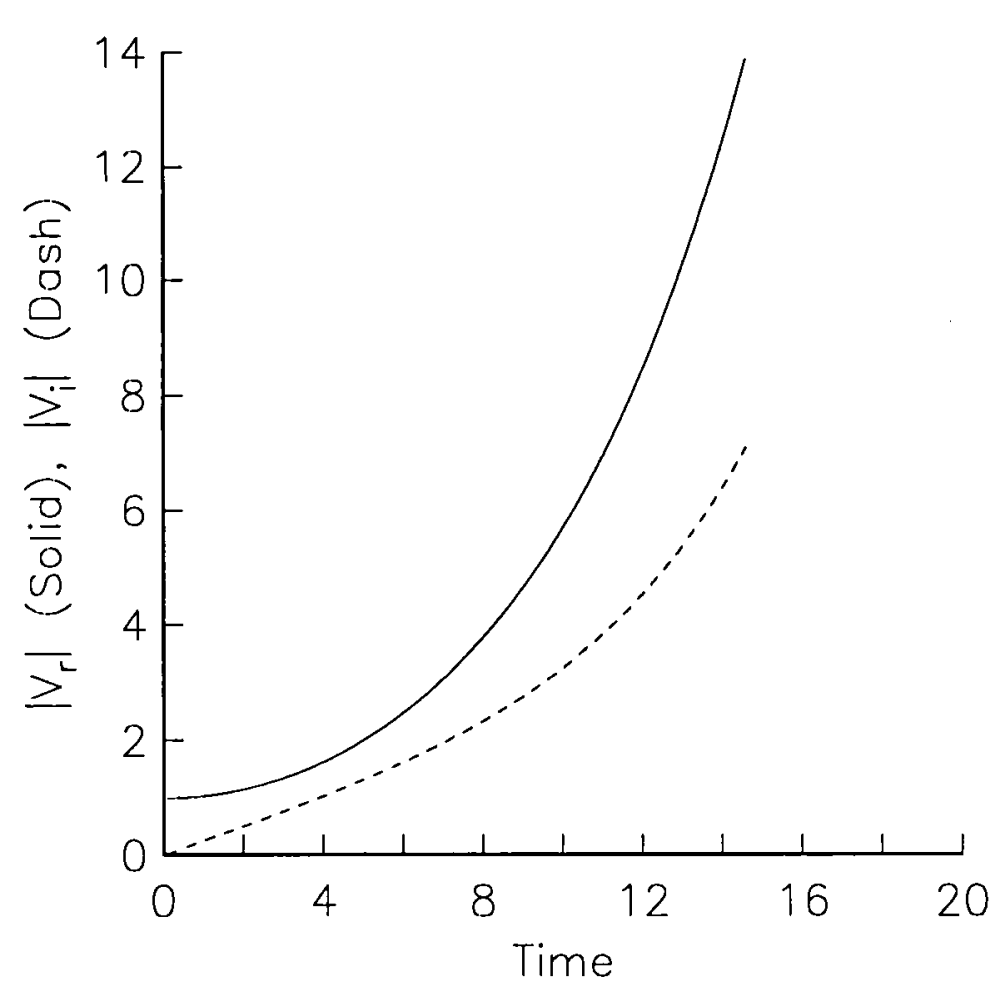

(A)

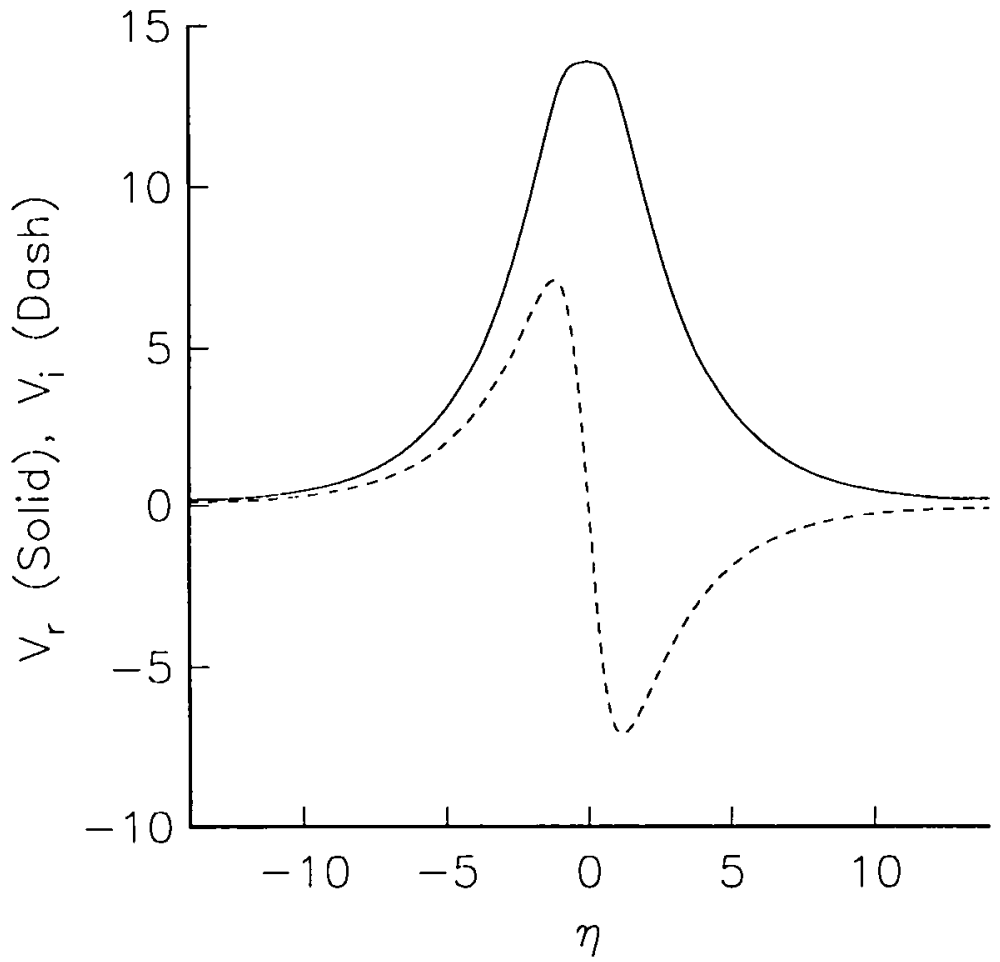

(B)

Figure 9. (A) Plot of the maximum magnitudes of real (solid) and imaginary (dashed) parts of $V$ as a function of time $T$ for asymmetric initial disturbance for $\phi=0, \alpha=0.396$ and $\beta_{0}=1$. (B) Plot of real (solid) and imaginary (dashed) parts of $V$ as a function of $\eta$ at $T=15.4$ for $\phi=0$ and $\beta_{0}=1$. 


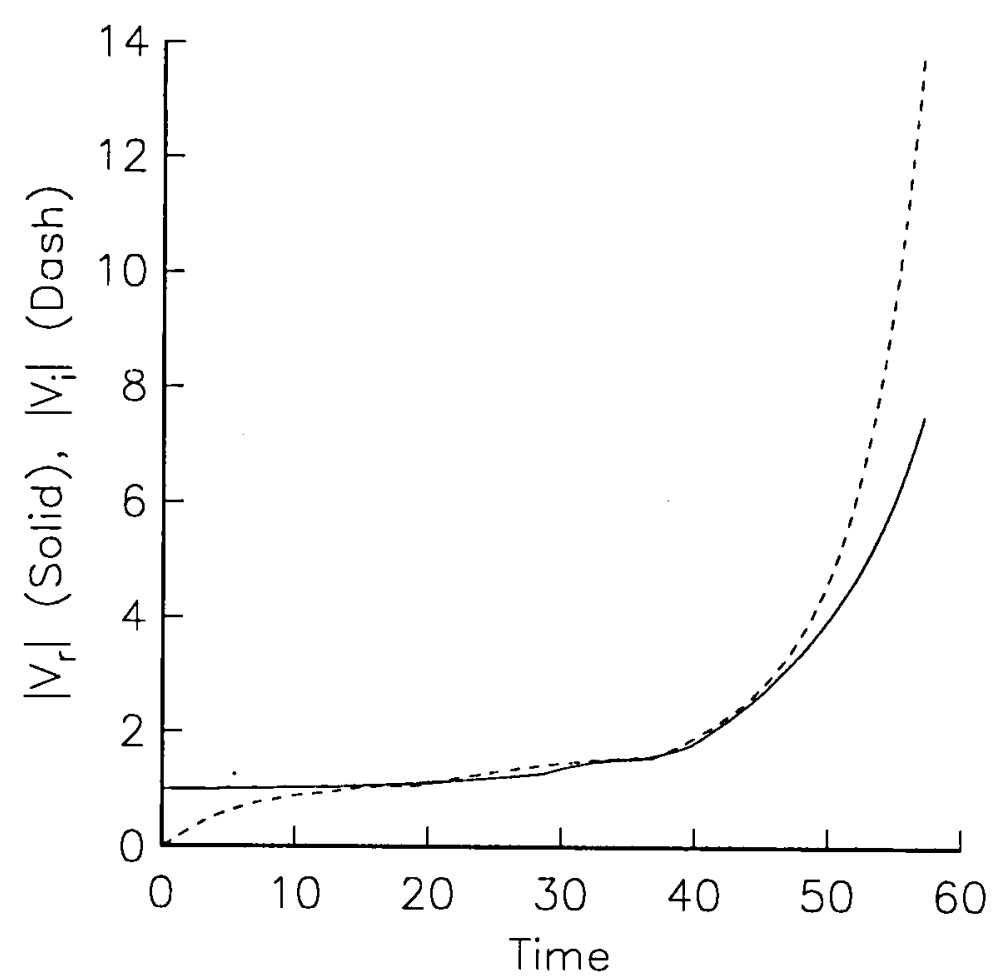

(A)

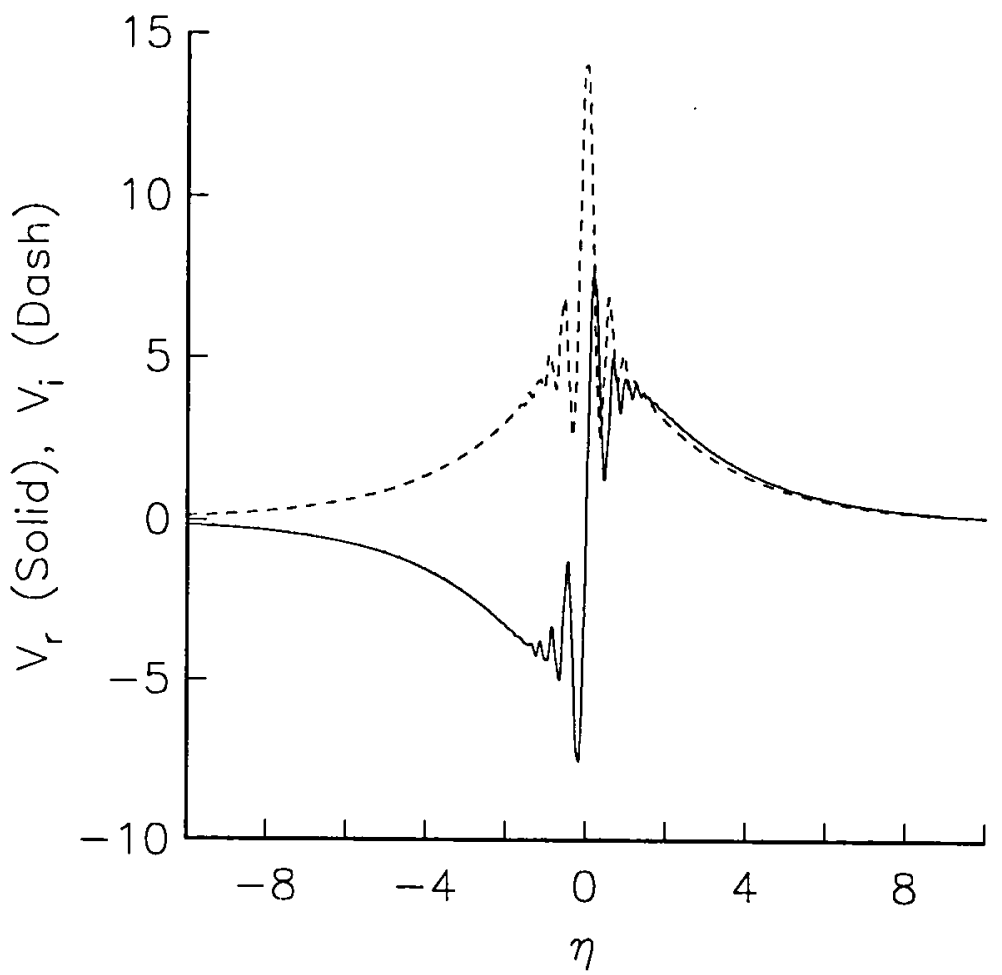

(B)

Figure 10. (A) Plot of the maximum magnitudes of real (solid) and imaginary (dashed) parts of $V$ as a function of time $T$ for asymmetric initial disturbance for $\phi=0, \alpha=0.396$ and $\beta_{0}=1$. (B) Plot of real (solid) and imaginary (dashed) parts of $V$ as a function of $\eta$ at $T=58$ for $\phi=0$ and $\beta_{0}=10 \pi$. 




REPORT DOCUMENTATION PAGE
$\begin{aligned} & \text { Rorm Approved } \\
& \text { OMB No. 0704-0188 }\end{aligned}$
$\begin{aligned} & \text { Public reporting burden for this collection of information is estimated to average } 1 \text { hour per response, including the time for reviewing instructions, searching existing data sources, } \\
& \text { gathering and maintaining the data needed, and completing and reviewing the collection of information. Send comments regarding this burden estimate or any other aspect of this } \\
& \text { collection of information, including suggestions for reducing this burden, to Washington Headquarters Services, Directorate for Information Operations and Reports, 1215 Jefferson } \\
& \text { Davis Highway, Suite 1204. Arlington, VA 22202-4302, and to the Office of Management and Budget. Paperwork Reduction Project (0704-0188). Washington, DC 20503. }\end{aligned}$
\begin{tabular}{|l|l|l|l|} 
1. AGENCY USE ONLY(Leave blank) & 2. REPORT DATE \\
June 1994 & 3. REPORT TYPE AND DATES COVERED \\
Contractor Report
\end{tabular}

4. TITLE AND SUBTITLE

TOWARDS ENHANCING AND DELAYING DISTURBANCES IN

FREE SHEAR FLOWS

6. AUTHOR(S)

W.O. Criminale

T.L. Jackson

D.G. Lasseigne

7. PERFORMING ORGANIZATION NAME(S) AND ADDRESS(ES)

Institute for Computer Applications in Science

and Engineering

Mail Stop 132C, NASA Langley Research Center

5. FUNDING NUMBERS

C NAS1-19480

WU 505-90-52-01

Hampton, VA 23681-0001

9. SPONSORING/MONITORING AGENCY NAME(S) AND ADDRESS(ES)

National Aeronautics and Space Administration

Langley Research Center

Hampton, VA 23681-0001

8. PERFORMING ORGANIZATION

REPORT NUMBER

ICASE Report No. 94-56

\section{SUPPLEMENTARY NOTES}

Langley Technical Monitor: Michael F. Card

Final Report

Submitted to Journal of Fluid Mechanics

12a. DISTRIBUTION/AVAILABILITY STATEMENT

Unclassified-Unlimited

Subject Category 34

13. ABSTRACT (Maximum 200 words)

The family of shear flows comprising the jet, wake, and the mixing layer are subjected to perturbations in an inviscid, incompressible fluid. By modeling the basic mean flows as parallel with piecewise linear variations for the velocities, complete and general solutions to the linearized equations of motion can be obtained in closed form as functions of all space variables and time when posed as an initial-value problem. The results show that there is a continuous as well as the discrete spectrum that is more familiar in stability theory and therefore there can be both algebraic and exponential growth of disturbances in time. These bases make it feasible to consider control of such flows. To this end, the possibility of enhancing the disturbances in the mixing layer and delaying the onset in the jet and wake is investigated. It is found that growth of perturbations can be delayed to a considerable degree for the jet and the wake but, by comparison, cannot be enhanced in the mixing layer. By using moving coordinates, a method for demonstrating the predominant early and long time behavior of disturbances in these flows is given for continuous velocity profiles. It is shown that the early time transients are always algebraic whereas the asymptotic limit is that of an exponential normal mode. Numerical treatment of the new governing equations confirm the conclusions reached by use of the piecewise linear basic models. Although not persued here, feedback mechanisms designed for control of the flow could be devised using the results of this work.

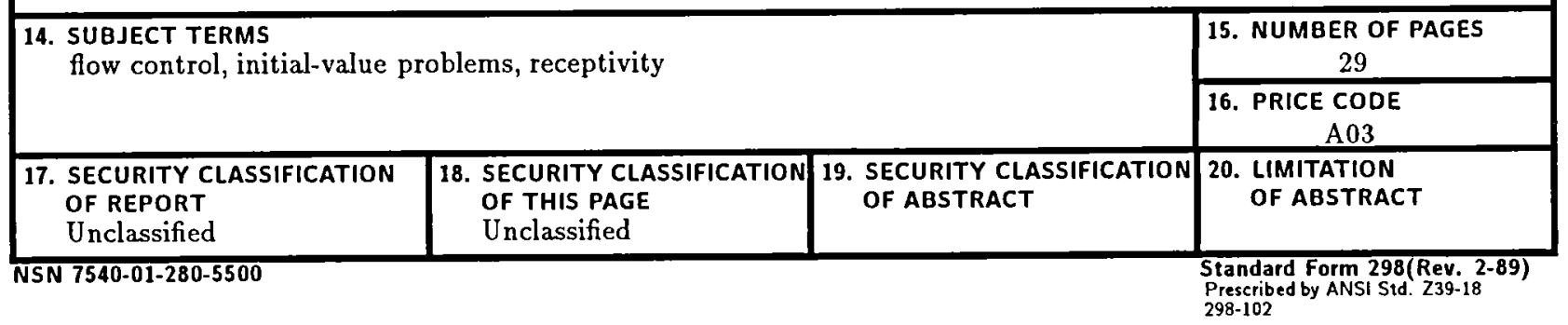



National Aeronautics and

Space Administration

Langley Research Center

Mail Code 180

Hampton, VA 23681-00001

BULK RATE

POSTAGE \& FEES PAID

NASA

Orficlal Business

Permit No. G-27

Penalty for Privato Use, $\$ 300$

NASA Technical Libra

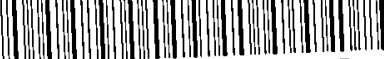

31176014131263 